PREPARED FOR THE U.S. DEPARTMENT OF ENERGY, UNDER CONTRACT DE-AC02-76CH03073

PPPL-3997

PPPL-3997

UC-70

The Frequency and Damping of Ion Acoustic Waves in Collisional and Collisionless Two-species Plasma

by

R.L. Berger and E.J. Valeo

August 2004

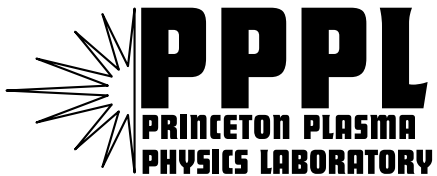

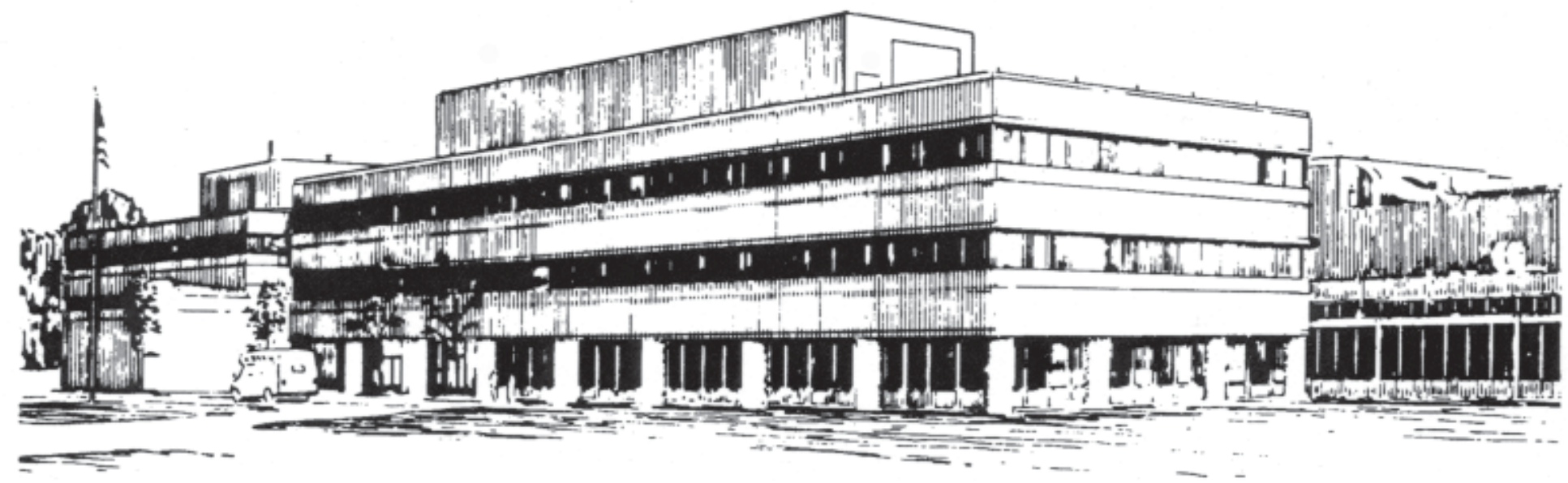

PRINCETON PLASMA PHYSICS LABORATORY PRINCETON UNIVERSITY, PRINCETON, NEW JERSEY 


\section{PPPL Reports Disclaimer}

This report was prepared as an account of work sponsored by an agency of the United States Government. Neither the United States Government nor any agency thereof, nor any of their employees, makes any warranty, express or implied, or assumes any legal liability or responsibility for the accuracy, completeness, or usefulness of any information, apparatus, product, or process disclosed, or represents that its use would not infringe privately owned rights. Reference herein to any specific commercial product, process, or service by trade name, trademark, manufacturer, or otherwise, does not necessarily constitute or imply its endorsement, recommendation, or favoring by the United States Government or any agency thereof. The views and opinions of authors expressed herein do not necessarily state or reflect those of the United States Government or any agency thereof.

\section{Availability}

This report is posted on the U.S. Department of Energy's Princeton Plasma Physics Laboratory Publications and Reports web site in Fiscal Year 2004. The home page for PPPL Reports and Publications is: http://www.pppl.gov/pub_report/

DOE and DOE Contractors can obtain copies of this report from:

U.S. Department of Energy

Office of Scientific and Technical Information

DOE Technical Information Services (DTIS)

P.O. Box 62

Oak Ridge, TN 37831

Telephone: (865) 576-8401

Fax: (865) 576-5728

Email: reports@adonis.osti.gov

This report is available to the general public from:

National Technical Information Service

U.S. Department of Commerce

5285 Port Royal Road

Springfield, VA 22161

Telephone: $1-800-553-6847$ or

(703) $605-6000$

Fax: (703) 321-8547

Internet: http://www.ntis.gov/ordering.htm 


\title{
The frequency and damping of ion acoustic waves in collisional and collisionless two-species plasma
}

\author{
R. L. Berger* \\ University of California, Lawrence Livermore national Laboratory, \\ P. O. Box 808, Livermore, CA 94551 \\ E. J. Valeo \\ Princeton Plasma Physics Laboratory, Princeton, NJ 08540
}

(Dated: July 14, 2004)

\begin{abstract}
The dispersion properties of ion acoustic waves (IAW) are sensitive to the strength of ion-ion collisions in multi-species plasma in which the different species usually have differing charge-tomass ratios. The modification of the frequency and damping of the fast and slow acoustic modes in a plasma composed of light (low Z) and heavy (high Z) ions is considered. In the fluid limit where the light ion scattering mean free path, $\lambda_{l h}$ is smaller than the acoustic wavelength, $\lambda=2 \pi / k$, the interspecies friction and heat flow carried by the light ions scattering from the heavy ions causes the damping. In the collisionless limit, $k \lambda_{l h}>>1$, Landau damping by the light ions provides the dissipation. In the intermediate regime when $k \lambda_{l h} \sim 1$, the damping is at least as large as the sum of the collisional and Landau damping.
\end{abstract}

\footnotetext{
${ }^{*}$ Electronic address: berger5@llnl.gov
} 


\section{INTRODUCTION}

Ion acoustic waves in magnetic-field-free plasmas play an essential role in the evolution of many important processes such as laser-plasma scattering instabilities, return current instabilities, and in the nonlinear stability of plasma waves. Of particular interest are the dispersive properties of ion acoustic waves in high-temperature, laser-produced plasmas created in experiments on large laser facilities with $40-200 \mathrm{~kJ}$ and focused laser intensities in excess of $10^{13} \mathrm{~W} / \mathrm{cm}^{2}$ and smaller laser facilities with $\sim 100 \mathrm{~J}$ and focused laser intensities less than $10^{16} \mathrm{~W} / \mathrm{cm}^{2}$.[1] The vast majority of laser-plasma experiments use solid paralene $(\mathrm{CH})$ or hydrocarbon gases such as propane $\left(\mathrm{C}_{3} \mathrm{H}_{8}\right)$ as a target material that is quickly ionized to make $0.5-4 \mathrm{keV}$ electron temperature plasma at electron densities of $1-100 \times 10^{19} \mathrm{~cm}^{-3}$. One experiment, specially designed to probe the properties of ion acoustic waves in a low-Z, high-Z mixture of gold and beryllium,[2] was motivated in part on the basis of collisionless theory of ion acoustic waves in multiple-species plasmas.[3],[4] Thomson scatter from the ion waves in a gold-beryllium plasma has been used as an ion temperature diagnostic. [5] High-Z gases, such as argon or xenon, have been added to low-Z plasma for spectroscopic diagnosis or to create X-ray sources. In some experiments, the addition of "trace" amounts of high-Z diagnostic dopants appears to affect the results.[6] More recently, experiments have addressed specifically the dependence of the stimulated scattering from ion acoustic waves on plasma charge state, $Z$, both for pure gases and for small amounts of high $Z$ in hydrocarbon plasma.[7]

For a plasma of more than one ion species, the acoustic wave frequency dispersion of a single-ion-species plasma is easily generalized to $\omega_{f}=k \lambda_{d e} \sum_{j} \omega_{p i, j}$ where $\lambda_{d e}$ is the electron Debye length and, for species $j$, the ion plasma frequency, $\omega_{p i, j}=\sqrt{4 \pi N_{j}^{2} e^{2} Z_{j}^{2} / m_{j}}$. This dispersion assumes that the phase velocity, $\omega_{f} / k v_{j}>>1$ for all species, $j$. The notation, $\omega_{f}$, where the subscript stands for "fast", anticipates the existence of other acoustic modes where the $\omega_{f} / k v_{j}<1$ for at least one species. Consider the case of two ion species and assume a mode exists such that the phase velocity lies between the lighter and heavier ion thermal velocity. That is, the lighter ions play for this "slow" mode a role similar to the role the electrons play for the fast wave. Such modes are well-known.[3, 4],[8]-[12]

In addition to the practical applications, the theory of acoustic waves in multiple-species plasma is inherently interesting problem in plasma physics. There have been numerous con- 
tributions to the theory of multiple-species ion waves within the context of the hydrodynamic equations[8],[9] or the Vlasov equation coupled with Maxwell's equations.[3],[4],[10],[11],[12] The theory presented here is (to our knowledge) the first to consider the general case where both interspecies collisions and Landau damping are treated. The hydrodynamic equations are valid in the limit that the self-collisions and interspecies collisions are frequent enough to maintain an isotropic Maxwellian; that is the ion-ion mean free path, $\lambda_{i j}=V_{i} / \nu_{i, j}$ (where $\left.\nu_{i, j}\left(V_{i}\right) \sim 4 \pi Z_{i}^{2} Z_{j}^{2} e^{4} N_{j} \ln \left(\Lambda_{i j}\right) / m_{i}^{2} V_{i}^{3}\right)$ is much less than the acoustic wavelength and the acoustic frequency $\omega$ is much less than the ion-ion collision rate, $\nu_{i, j}$. The assumption here is that the relative velocity is the light ion velocity with thermal velocity, $V_{i}=\sqrt{T_{i} / m_{i}}$. In the limit that both these inequalities are reversed, the Vlasov equation is the appropriate description. The acoustic wavelengths that motivate this work are set by laser plasma interactions (such as stimulated Brillouin scattering, filamentation, or Thomson scatter) which scale with the laser wavelength, namely, $351-1053 \mathrm{~nm}$. Because the wavenumber of the acoustic wave that backscatters the laser light is $10-50 \times$ the wavenumber for forward scatter, one approximation is unlikely to be appropriate in all cases of interest.

This problem is similar to acoustic wave dispersive properties in a single species plasma when the electron-ion mean free path is comparable to the acoustic wavelength.[13],[14] In fact, we adopt the methods developed for that problem by using the linearized FokkerPlanck equation for the light ion with a Lorentz collision operator to account for interspecies collisions.[13] Given the strong self-collisions of the higher $Z$ species, we treat them as a cold fluid.

We present explicit results of the frequency and damping of slow and fast ion acoustic waves for the example of a mixture of hydrogen and xenon. The $[Z=40, A=131]$ xenon concentration is varied from $1 \%$ to $10 \%$ by atom. The Lorentz gas approximation to the interspecies collision operator made in deriving the linearized Fokker-Planck equation is more accurate for heavy, high-Z ions such as gold or xenon than lighter, lower-Z ions such as carbon. In addition, the frequencies of the slow and fast waves are distinctly separated for mixtures of xenon and hydrogen which allows for unambiguous Thomson scatter signatures. We find that the dispersive properties of the waves are greatly modified by interspecies (light-heavy) collisions when $|\omega| \lesssim \nu_{l h}$ and $k \lambda_{l h} \lesssim 1$ where

$$
\nu_{l h}=\frac{4 \pi Z_{l}^{2} Z_{h}^{2} e^{4} N_{h} \ln \left(\Lambda_{l h}\right)}{m_{l}^{2} V_{l}^{3}}
$$




$$
\lambda_{l h}=\frac{V_{l}}{\nu_{l h}}=\frac{T_{i}^{2}}{4 \pi Z_{l}^{2} Z_{h}^{2} e^{4} N_{h} \ln \left(\Lambda_{l h}\right)}
$$

The first inequality means that the (higher-frequency) fast wave dispersion remains Vlasovlike for a larger $\nu_{l h}$ than the slow wave. Also because the heavy ions oscillate nearly out of phase with the light ions in the slow wave, the slow wave does not exist as a normal mode in semi-collisional limit, $k \lambda_{l h} \sim 0.1$. The fast wave, which exists for all $k \lambda_{l h}$, is Landau damped when the phase velocity, $\omega / k$, approaches the light ion thermal velocity, $V_{l}$ (high ion temperature and large $k \lambda_{l h}$ ) and collisionally damped for $\omega / k V_{l}>>1$ (low ion temperature and small $k \lambda_{l h}$ ). Application of the theory to hydrocarbon plasma with typical parameters of Inertial Fusion Experiments shows significant modification of the dispersive properties as well. In experiments where the acoustic modes of a Be-Au plasma were probed with Thomson scatter[2], $k \lambda_{l h} \sim 3$ for a $1 \% \mathrm{Au}$ and $k \lambda_{l h} \sim 0.5$ for a $10 \% \mathrm{Au}$; in this case, interspecies collisions may affect the interpretation of the Thomson scatter as we discuss in Sec. IV.

In Sec. II, the dispersion relations are obtained in turn from the linearized fluid, collisionless (Vlasov), and Fokker-Planck equations. In Sec. III. the frequency and damping of slow and fast ion acoustic waves in two species plasmas are found as a function of the ion-ion mean free path as determined by the ion temperature variation for fixed wavelength or by the acoustic wavelength variation for fixed ion temperature. In Sec. IV, we conclude.

\section{TWO SPECIES ION ACOUSTIC WAVES}

The frequency and damping of ion acoustic waves in multiple species plasmas has been examined previously in the collisionless limit where the Landau damping prevails and in the hydrodynamic limit where interspecies friction and viscosity prevail.[8],[9] In the collisionless limit [3],[4], the coupling between species occurs only through the self-consistent electric field, and the general dispersion relation for any number of ion species is easily obtained. When collisions between species are important, the calculation is tedious beyond two species if the collisional coupling between all species is retained. If the different species have different charge-to-mass ratios, interspecies friction, in the collisional limit defined by $k \lambda_{i j}<<1$ for all species $\mathrm{i}, \mathrm{j}$, is the dominant dissipation because the ions accelerate to different oscillatory velocities in the electric field. In plasmas consisting of heavy, high $\mathrm{Z}$ (e.g. Au or Xe) 
materials mixed with light, low-Z materials (e.g. H or Be), the heavy species distribution is constrained to be an isotropic Maxwellian because of the strong self collisions but the light species collisionality relies on the inter-species scattering, somewhat like the scattering of electrons from ions. In one limit where the number of high-Z and low-Z ions is similar, the frequency of the mode is determined by the heavy ions $\left(N_{l} Z_{l}^{2} / A_{l}<<N_{h} Z_{h}^{2} / A_{h}\right)$ but the dissipation depends on the number of light ions. In another limit where the heavy species is a very small fraction, the mode frequency is determined by light ions but the heavy ions still dominant the collision rate (because $N_{l} Z_{l}^{2}<<N_{h} Z_{h}^{2}$ ).

In light of the dominance of inter-species collisions, we consider the Fokker-Planck equation for the light ions in which the self-collisions are kept only in the lowest order but the collisions from the heavy ions are kept to all orders. The light ion distribution, $f_{l}$, is expanded according to,

$$
f_{l}=F_{l}+\delta f_{l}
$$

where $F_{l}$, an isotropic Maxwellians with density $N_{l}$ and temperature $T_{l}$, satisfies the lowest order equation. The evolution of the light ion perturbed distribution function (in the frame oscillating with the light ions) is governed by the equation,

$$
\left(\frac{\partial}{\partial t}+v \cdot \nabla\right) \delta f_{l}+\left(\frac{e Z_{l}}{m_{l}} E-\frac{\partial U_{l}}{\partial t}\right) \cdot \frac{\partial}{\partial v} F_{l}-\frac{\partial}{\partial x_{\alpha}} U_{\beta} v_{\alpha} \frac{\partial}{\partial v_{\beta}} F_{l}=C_{l h}\left(\delta f_{l}, F_{h}\right) .
$$

where $F_{h}$ is the isotropic Maxwellian of the heavy ions with density $N_{h}$ and temperature $T_{h}$. If the heavy and light ions have similar temperature, the relative velocity in a collision is to a good approximation the light ion velocity. Thus, the collisional interaction of light ions with heavy ions can be treated in a manner similar to the collisions of electrons from ions. One result is that the light-heavy collisions act to isotropize the light ion distribution. Unlike the single-ion-species, electron-ion plasma where quasi-neutrality requires, $U_{e}=U_{i}$, where $U_{e, i}$ is the electron, ion flow velocity, the light and heavy ion flow velocities, $U_{l}$ and $U_{h}$, are generally unequal. As shown by Epperlein[8], the right hand light of Eq. (4) can be written,

$$
C_{l h}=-\nu_{1}(v)\left[\frac{\partial}{\partial \mu}\left(1-\mu^{2}\right) \frac{\partial}{\partial \mu} \delta f_{l}+\left(U_{h}-U_{l}\right) \cdot \frac{\partial}{\partial v} F_{l}\right]
$$

where $\mu=(v \cdot E /(v E))$ and $F_{l}$ is the isotropic distribution of the light ions in the light ion frame. Again, the rate of collisions of the light ions from the heavy ions is,

$$
\nu_{1}(v)=\frac{4 \pi Z_{l}^{2} Z_{h}^{2} e^{4} N_{h} \ln \left(\Lambda_{l h}\right)}{m_{l}^{2} v^{3}} .
$$




\section{A. The hydrodynamic limit}

In the limit that $k \lambda_{i j}<<1$ for all species $\mathrm{i}, \mathrm{j}$, the fluid equations are appropriate. Expanding $\delta f_{l}$ in a Legendre polynomial series but keeping only the $l=0,1$ coefficients allows a solution for $\delta f_{l, 1}$ in terms of $F_{l}$ and $f_{l, 0}$. The set of fluid moment equations for the density, momentum, and energy generated by integrating Eq. (4) with weights $\left(1, m_{l} v \mu,(1 / 2) m_{l} v^{2}\right)$ is closed with the friction force and the heat flow determined by moments of $\delta f_{l, 1}$. The linearized particle conservation equations for each species are,

$$
-i \omega n_{j}+i k N_{j} U_{j}=0, \text { for } j=l, h
$$

For the light ions, the momentum evolves according to,

$$
-i \omega N_{l} m_{l} U_{l}=e Z N_{l} E-i k T_{l} n_{l}-i k N_{l} \delta T_{l}+R_{l h}
$$

where the friction force, $R_{l h}$ is $[8]$,

$$
R_{l h}=-\alpha_{0} N_{l} m_{l} \frac{\left(U_{l}-U_{h}\right)}{\tau_{l h}}-i k \beta_{0} N_{l} \delta T_{l} .
$$

The heavy ion momentum evolves according to,

$$
-i \omega N_{h} m_{h} U_{h}=e Z_{h} N_{h} E-i k T_{h} n_{h}-i k N_{h} \delta T_{h}+R_{h l}
$$

where $R_{h l}=-R_{l h}$ and the collision time of light ions from heavy ions is, $\tau_{l h}=(\sqrt{2 / \pi} / 3) / \nu_{l h}$, where $\nu_{l h}=\nu_{1}\left(V_{l}\right)$ given in Eq. (6) and the light ion thermal velocity is $V_{l}=\sqrt{T_{l} / m_{l}}$. The light ion temperature evolves according to,

$$
-i \omega \beta_{0} N_{l} \delta T_{l}+i k N_{l} T_{l} U_{l}=-i k q_{l},
$$

where

$$
q_{l}=-i k K_{l h} \delta T_{l}+\beta_{0} N_{l} T_{l}\left(U_{l}-U_{h}\right),
$$

and $K_{l h}=\gamma_{0} N_{l} T_{l} \tau_{l h} / m_{l}, \beta_{0}=3 / 2, \alpha_{0}=3 \pi / 32$, and $\gamma_{0}=128 / 3 \pi$. The heavy ion heat flow is negligible and the heavy ions obey the adiabatic temperature equation,

$$
-i \omega \beta_{0} N_{h} \delta T_{h}+i k N_{h} T_{h} U_{h}=0,
$$

The electrons are in pressure balance with the electric field, E.

$$
-i k T_{e} n_{e}-e N_{e} E=0
$$


Because the ion scattering mean free path can be of the same magnitude as the electron Debye length for high Z plasma (unlike the electron-ion collisional effects on the electron damping of ion acoustic waves where $\lambda_{e i}>>\lambda_{d e}$ ), quasi-neutrality may not be a good approximation. Even for rather small fractions by atom of heavy ions, $Z_{h} N_{h} \sim N_{e}$, with the consequence that the heavy ion Debye length is much smaller than the electron Debye length, $\lambda_{D h} / \lambda_{D e} \sim \sqrt{T_{i} / Z_{h} T_{e}}<<1$. No general statement of the ratio of the light ion Debye length to the electron Debye length is possible. With allowance for charge separation with Poisson's equation,

$$
i k E=4 \pi e\left(\sum_{j=l, h} Z_{j} n_{j}-n_{e}\right),
$$

the dispersion relation can be expressed in the form:

$$
\begin{array}{r}
1-\frac{1}{i \omega\|D\|}\left(\omega_{a, l l}^{2} D_{h h}+\omega_{a, h h}^{2} D_{l l}-\omega_{a, h l}^{2} D_{l h}-\omega_{a, l h}^{2} D_{h l}\right)=0, \\
D_{l l}=-i \omega+k^{2} V_{l}^{2}\left(\frac{i}{\omega}+\frac{25}{4} \frac{1}{\Omega}\right)+\frac{\alpha_{0}}{\tau_{l h}}, \\
D_{h h}=-i \omega+\frac{5 i}{3} \frac{k^{2} V_{h}^{2}}{\omega}+\frac{9}{4} r_{l h} \frac{k^{2} V_{l}^{2}}{\Omega}+r_{l h} \frac{\alpha_{0}}{\tau_{l h}}, \\
D_{l h}=-\left(\frac{\alpha_{0}}{\tau_{l h}}+\frac{15}{4} \frac{k^{2} V_{l}^{2}}{\Omega}\right) \\
D_{h l}=r_{l h} D_{l h}, \\
\Omega=-\frac{3}{2} i \omega+\gamma_{0} k^{2} V_{l}^{2} \tau_{l h},
\end{array}
$$

where $\mathbf{D}$ is the matrix that relates the flow velocity vector $\mathbf{U}=\left[U_{l}, U_{h}\right]$ to the electric field with determinant,

$$
\|\mathbf{D}\|=D_{l l} D_{h h}-D_{l h} D_{h l},
$$

and the partial acoustic frequencies,

$$
\omega_{a, j j^{\prime}}^{2}=\left(1+k^{2} \lambda_{d e}\right)^{-1} \frac{k^{2} T_{e} Z_{j} Z_{j^{\prime}} N_{j^{\prime}}}{N_{e} m_{j}},
$$

and the ratio of the mass density, $r_{l h}=m_{l} N_{l} / m_{h} N_{h} ; r_{l h} \sim 1$ for dilute mixtures of high $Z$ material. We neglect the electron contribution to the IAW damping from collisional or collisionless processes[13].

In the long wavelength or low ion temperature limit where $\omega \gg k V_{l} \gg k V_{h}$, the terms in Eqs.17,18,19, and 20 that originate from the ion pressure gradients are small compared to the drag and frequency. In that limit, the dispersion relation is a complex cubic polynomial. 
Assuming a root with $\Im(\omega) \ll \Re(\omega)$, one finds the approximate solution,

$$
\begin{aligned}
\omega_{r}^{2}=\omega_{f}^{2} & +2 \triangle_{1} \omega_{i}, \\
\omega_{i} & =\frac{\triangle_{2}-\triangle_{1} \omega_{f}^{2}}{2\left(\omega_{f}^{2}+\triangle_{1}^{2}\right)}
\end{aligned}
$$

where

$$
\begin{aligned}
\omega_{f}^{2} & =\omega_{a, l l}^{2}+\omega_{a, h h}^{2}, \\
\triangle_{1} & =\frac{\left(1+r_{l h}\right) \alpha_{0}}{\tau_{l h},} \\
\triangle_{2} & =\frac{\left(r_{l h} \omega_{a, l l}^{2}+\omega_{a, h h}^{2}+2 \omega_{a, l h}^{2}\right) \alpha_{0}}{\tau_{l h} .}
\end{aligned}
$$

Because $\triangle_{1} \gg \omega_{f}$, the damping is weak and proportional to $\tau_{l h}$ in this limit. No slow mode roots are found in this limit but a weakly damped non-oscillatory mode is found. The damping is weak for the fast wave because the strong friction forces the heavy and light ions to the same velocity. On the other hand, for the slow mode to exist, the heavyion and light-ion oscillatory velocities must have the opposite signs which is precluded by strong friction. For higher ion temperature or shorter wavelength, the collision rate, thus the friction, decreases. The off-diagonal components of $\mathbf{D}$ are small and the diagonal components are in first approximation equal to $-i \omega$. To lowest order in $k \lambda_{l h}$, the fast wave solution to Eq. (16) is again,

$$
\omega_{f}^{2}=\omega_{a, l l}^{2}+\omega_{a, h h}^{2}
$$

and the damping appears in the next order proportional to $\tau_{l h}^{-1}$. Here, the dominant contribution to the fast wave damping is caused by the thermal heat conduction of the light ions.

The phase velocity of the slow mode is between the light and heavy thermal velocity. Without the ion pressure contribution, the slow frequency, $\omega_{s}=0$. The mode exists in the fluid limit only when $k V_{l} \tau_{l h} \gtrsim 1$, and then the thermal conduction damping is more important than the explicit drag as was the case for the fast wave discussed in the previous paragraph. If the ratio of the heavy-to-light masses is large, the mode frequency is given by,

$$
\omega_{s}^{2}=\frac{\omega_{h h}^{2} k^{2} V_{l}^{2}}{\left(\omega_{l l}^{2}+k^{2} V_{l}^{2}\right)},
$$

which shows that the phase velocity is a nearly constant fraction of the light ion thermal velocity provided the light ion Debye length is much less than the wavelength, $V_{l} / \omega_{l l} \ll k^{-1}$. 
As an example, we consider the acoustic modes of a mixture that is $99 \%$ hydrogen atoms and $1 \%$ xenon atoms ionized to $Z_{X e}=40$ at an electron density, $N_{e}=9 \times 10^{20} \mathrm{~cm}^{-3}$ and at an electron temperature, $T_{e}=2 \mathrm{keV}$. The acoustic wave wavelength is held fixed at $175.66 \mathrm{~nm}$ which corresponds to the wavelength that backscatters a light wave of wavelength, 351nm. The scattering mean free path of the protons from the xenon ions is varied over several orders of magnitude by varying the ion temperature.

In Fig. 1, the frequency, damping, and phase velocity of the fast and slow modes obtained from the fluid dispersion are shown. The slow mode frequency is about $10 \times$ smaller than the fast wave. The damping rate for both waves decreases as $k \lambda_{l h}$ increases except at very small $k \lambda_{l h}$ where the damping of the fast wave has a maximum. For the convective growth rate and threshold of SBS, the ratio of the damping rate to the frequency, also shown in Fig. 1, is important. As asserted previously in this section, the damping rate is primarily

caused by thermal conduction for $k \lambda_{l h} \gtrsim 0.1$. A similar difference exists for the fast wave. There is no significant effect of the ion thermal conduction on either mode frequency.

\section{B. The Collisionless Dispersion}

The collisionless dispersion relation for two species is presented here to provide a comparison for the more general treatment to be presented in the next section. Because the RHS of Eq. (4) is zero in this approximation, it is convenient to work in a nonoscillatory frame for both ion species. The general dispersion relation is,

$$
\begin{aligned}
1+\sum_{j} \frac{T_{e} N_{j} Z_{j}^{2}}{\left(1+k^{2} \lambda_{d e}^{2}\right) T_{i} N_{e}} W\left(\zeta_{j}\right) & =0 \\
\zeta_{j} & =\frac{\omega}{\sqrt{2} k V_{j}} \\
W(\zeta) & =(1+\zeta Z(\zeta)) \\
Z(\zeta) & =\pi^{-1 / 2} \int_{-\infty}^{+\infty} d t \frac{\exp \left(-t^{2}\right)}{t-\zeta}
\end{aligned}
$$

The fast mode has $\Re \zeta_{j}>1$ for both species. As the ion temperature increases, there is an increasing number of light ions near the phase velocity of the mode and, consequently, the Landau damping increases. In the slow wave, $\Re \zeta_{l}<1$ and the damping of the slow wave increases (more slowly than the frequency) as the ion temperature increases even though its phase velocity decreases slightly. The collisionless damping is entirely determined by the 

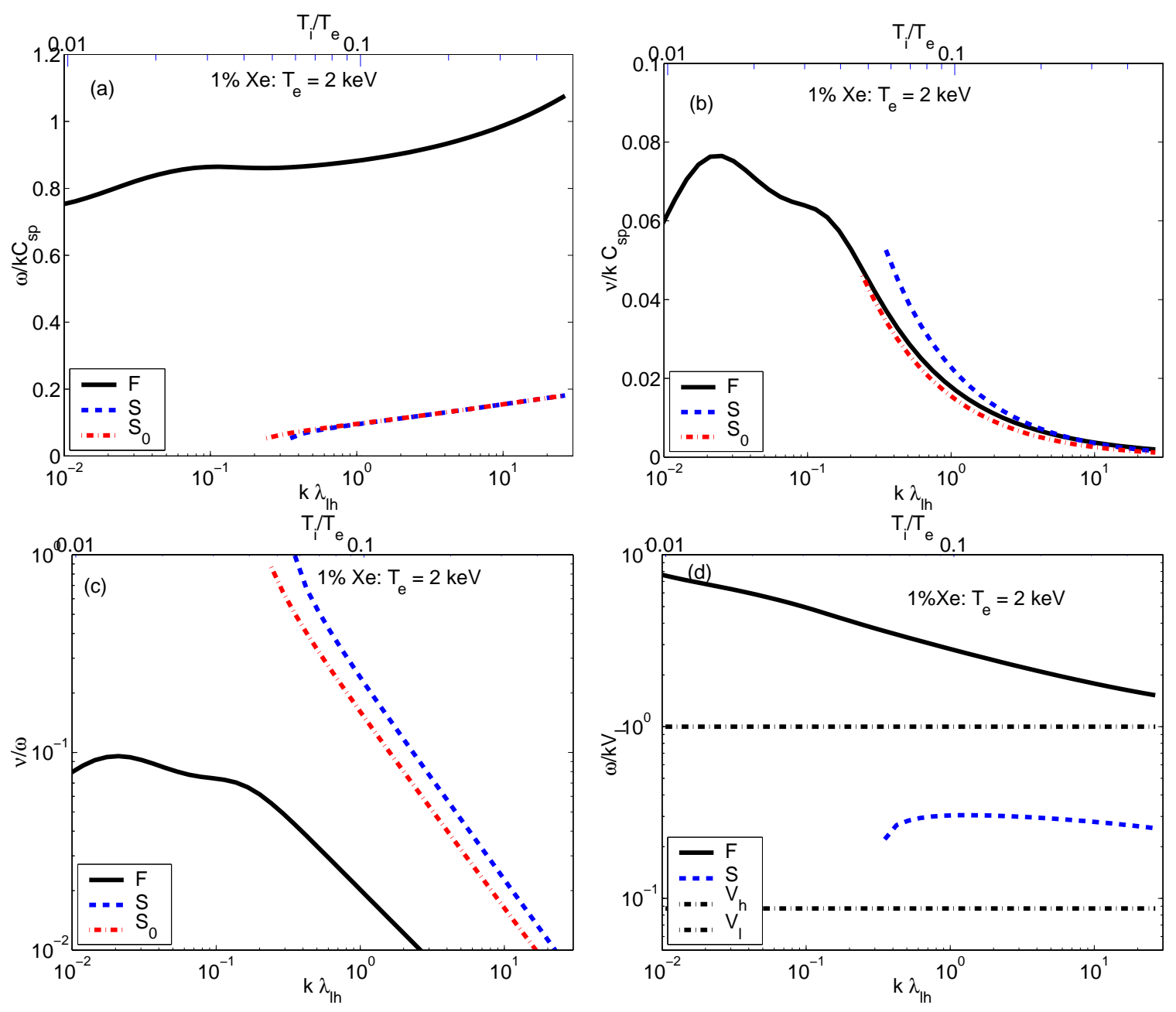

FIG. 1: The frequencies (a), damping rates (b) of the fast and slow acoustic waves normalized to the proton acoustic frequency, $k C_{s p} \equiv k \sqrt{T_{e} / m_{p}}$, the ratios of the damping rate to the frequency (c), and the phase velocities divided by the proton thermal velocity (d) are shown ( in the fluid approximation ) as a function of $k \lambda_{l h}$ for the fast wave $(\mathrm{F})$, the slow wave (S), and the slow wave without ion thermal conduction $\left(S_{0}\right)$. The top horizontal axis shows the value of $T_{i} / T_{e}$ for the values of $k \lambda_{l h}$ displayed on the bottom horizontal axis. In (d), the horizontal lines are the light ion thermal velocity $\left(V_{l}=1\right)$ and the heavy ion thermal velocity $\left(V_{h}=\sqrt{m_{l} / m_{h}}\right)$.

protons because $\Re \zeta_{h} \gg 1$. In Fig. 2 the frequency, Landau damping, and phase velocity of the slow and fast waves are shown as a function of $k \lambda_{l h} \sim T_{i}^{2}$ for the same parameters as those for Fig. 1. Of course, the collisionless dispersion cannot depend physically on $k \lambda_{l h}$; the collisionless frequency and damping are plotted versus $k \lambda_{l h}$ only to make comparison with the results that include collisions clearer. While the collisionless fast wave frequency 

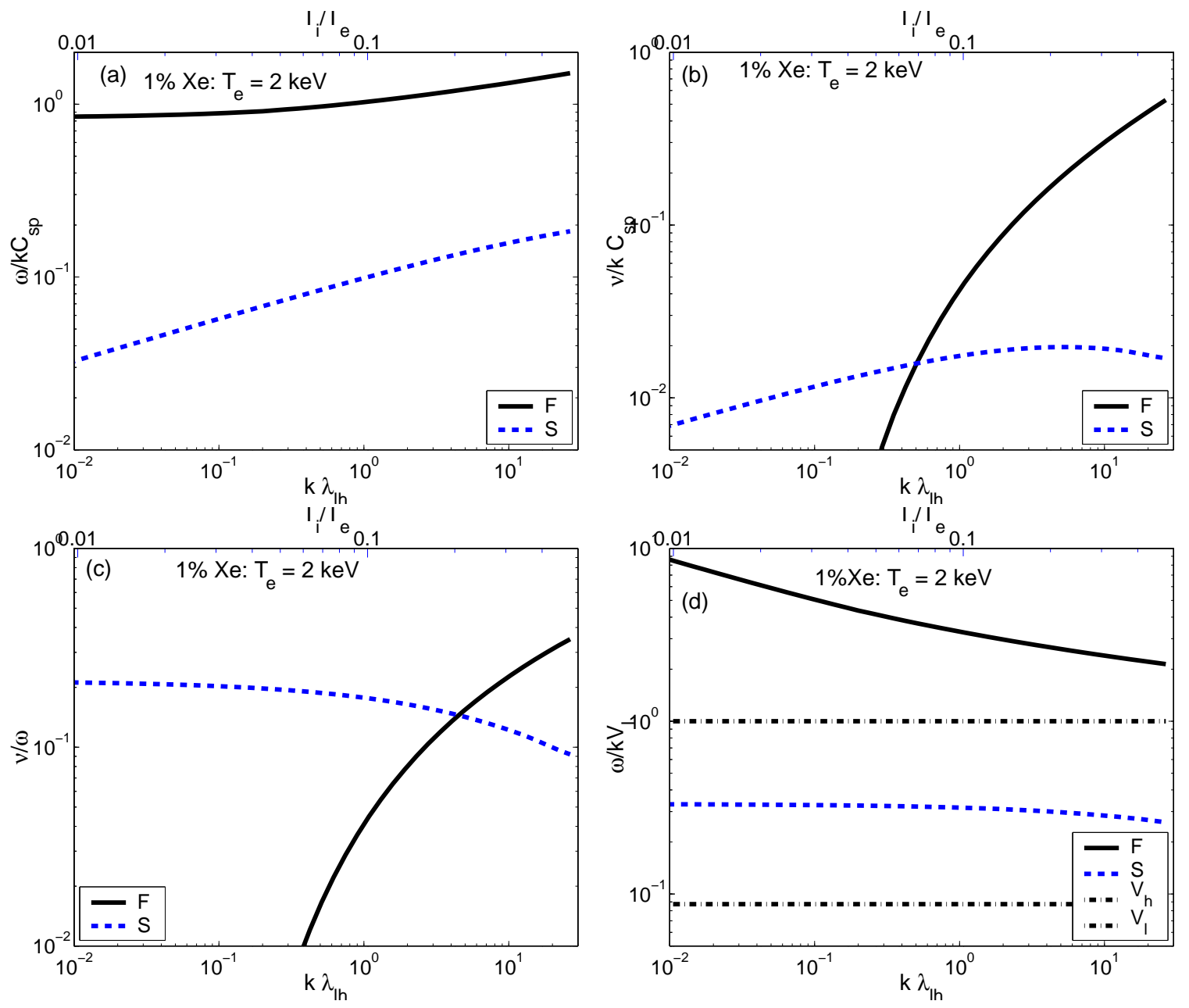

FIG. 2: The frequencies (a) and damping rates (b) normalized to the proton acoustic frequency $k C_{s p}$, the ratios of the damping rate to the frequency (c), and the phase velocities (d) of the fast (F) and slow (S) acoustic waves in the collisionless approximation are shown as a function of $k \lambda_{l h}$. The top horizontal axis shows the value of $T_{i} / T_{e}$ for the values of $k \lambda_{l h}$ displayed on the bottom horizontal axis. In (d), the horizontal lines, as in Fig. 1, are the hydrogen $\left(\mathrm{V}_{\mathrm{l}}\right)$ and xenon $\left(\mathrm{V}_{\mathrm{h}}\right)$ thermal velocities (normalized to the hydrogen thermal velocity).

in Fig. 2 depends weakly on the ion temperature, the slow wave frequency is proportional to $\sqrt{T_{i}}$ as is clear from the nearly constant value of $\omega / k V_{l}$ in Fig. 2 . Note that the Landau damping rate for both modes increases for $T_{i} / T_{e}>0.05$ whereas the collisional damping (shown in Fig. 1) decreased for both modes for $T_{i}>0.05 T_{e}=0.1 \mathrm{keV}$. The collisionless damping rate of the fast wave shown in Fig. 2 is very sensitive to the ion temperature and becomes the more strongly damped mode once $T_{i} / T_{e} \gtrsim 0.1$. The collisionless behavior is 
described in detail in Ref. [3, 4].

By comparing the results in the examples shown in 1 and 2, one notes that the predictions of the two are quite different. One expects and we will confirm subsequently that the fluid approximation applies for $k \lambda_{l h}<0.1$ and that the Vlasov approximation applies for $k \lambda_{l h}>10$. Now we address the behavior in the intermediate region where $0.1<k \lambda_{l h}<10$.

\section{The Fokker-Planck response for the light ions}

For two species, the light ion response can be found for arbitrary $k \lambda_{l h}$ by using the same methods used for finding the electron damping of ion acoustic waves for arbitrary $k \lambda_{e i}$ where the Lorentz collision operator is used for electron-ion collisions.[13] Of course, omitting terms proportional to $m_{l} / m_{h}$ compared to 1 is not as good as omitting ones proportional to $m_{e} / m_{i}$. Assuming a periodic perturbation of the form,

$$
\delta f_{l}=\sum_{j=0, \infty} f_{l, j}(v) P_{j}(\mu) \exp (-i(\omega t-k x))
$$

we generate from Eq. (4) the equations,

$$
\begin{aligned}
-i \omega f_{l, 0}+\frac{i k v}{3} f_{l, 1}-\frac{i k U_{l}}{3} v \frac{\partial}{\partial v} F_{l} & =0, \\
-i \omega f_{l, 1}+i k v f_{l, 0}+\frac{2 i k v}{5} f_{l, 2}+\left(\frac{e Z_{l}}{m_{l}} E+i \omega U_{l}\right) \frac{\partial}{\partial v} F_{l} & =C_{l h, 1}, \\
-i \omega f_{l, 2}+\frac{2 i k v}{3} f_{l, 1}+\frac{3 i k v}{7} f_{l, 3}-\frac{2}{3} i k v U_{l} \frac{\partial}{\partial v} F_{l} & =C_{l h, 2}, \\
-i \omega f_{l, j}+\frac{j}{2 j-1} i k v f_{l, j-1}+\frac{j+1}{2 j+1} i k v f_{l, j+1} & =C_{l h, j} .
\end{aligned}
$$

where Eq. (39) applies for $j>2$. The Lorentz collision operator (Eq. (5)) is diagonal for the Legendre polynomials with the result,

$$
C_{l h, j}=-\nu_{j} f_{l, j}+\delta_{j, 1} \nu_{1}(v)\left(U_{l}-U_{h}\right) \frac{\partial}{\partial v} F_{l}
$$

where $\nu_{j}=(j(j+1) / 2) \nu_{1}$. The heavy ions are described by the cold ion fluid equations.

$$
\begin{gathered}
-i \omega n_{h}+i k N_{h} U_{h}=0 \\
-i \omega N_{h} m_{h} U_{h}=e Z_{h} N_{h} E+R_{h l}
\end{gathered}
$$

Adopting the same method of continued fractions as used by Epperlein, one accounts for the $l>2$ Legendre coefficients $f_{l, j}$ by introducing the renormalized collision frequency $\nu_{l}^{*}$ 
such that

$$
i k v\left(\frac{l}{2 l-1}\right) f_{l, j-1}=-\nu_{l}^{*} f_{l, j},
$$

and one finds $f_{l, j=1}$ in terms of $U_{l, h}$ and $E$ :

$$
i k v f_{l, 0}-\left(\frac{-e Z_{l} E}{m_{l}}-i \omega U_{l}+\frac{4 k^{2} v^{2}}{15 \nu_{2}^{*}} U_{l}\right) \frac{\partial}{\partial v} F_{l}=-\nu_{1}^{*} f_{l, 1}+\nu_{1}\left(U_{l}-U_{h}\right) \frac{\partial}{\partial v} F_{l} .
$$

Using the relation between the renormalized collision frequencies (defined in Appendix A), $\nu_{2}^{*}$ and $\nu_{1}^{*}$,

$$
\frac{4 k^{2} v^{2}}{15 \nu_{2}^{*}}=\nu_{1}^{*}-\nu_{1}+i \omega
$$

one obtains from Eqs. 36 and 37,

$$
\begin{aligned}
f_{l, 0} & =\frac{i k v}{3 D}\left(\frac{e Z_{l} E}{m_{l}}+\nu_{1} U_{h}\right) \frac{\partial}{\partial v} F_{l} \\
f_{l, 1} & =\left(U_{l}+i \frac{\omega}{D}\left(\frac{e Z_{l} E}{m_{l}}+\nu_{1} U_{h}\right)\right) \frac{\partial}{\partial v} F_{l}, \\
& =\left(U_{l} \frac{\partial}{\partial v} F_{l}+\frac{3 \omega}{k v} f_{0}\right)
\end{aligned}
$$

where $D=-i \omega \nu_{1}^{*}+k^{2} v^{2} / 3$. The light ion perturbed density, $n_{l}$, and rate of momentum loss from the light ions to the heavy ions, $R_{l h}$, are obtained from $f_{l, 0}$ and $f_{l, 1}$ respectively with the relations,

$$
\begin{aligned}
n_{l} & =4 \pi \int_{0}^{\infty} d v v^{2} f_{l, 0} \\
R_{l h} & =-\frac{4 \pi m_{l}}{3} \int_{0}^{\infty} d v v^{3} \nu_{1}\left(f_{l, 1}+\left(U_{h}-U_{l}\right) \frac{\partial}{\partial v} F_{l}\right) .
\end{aligned}
$$

Because these equations are constructed in the light ion frame, $4 \pi / 3 \int_{0}^{\infty} d v v^{3} f_{l, 1}=0$, from which the light-ion continuity relation,

$$
N_{l} U_{l}=\frac{\omega}{k} n_{l}
$$

follows from Eq. (48). We define the electron and light,heavy ion susceptabilities as $\chi_{e}=$ $4 \pi e n_{e} / i k E$, and $\chi_{l, h}=-4 \pi e Z_{l, h} n_{l, h} / i k E$. Using $R_{h l}=-R_{l h}$ and eliminating $U_{l, h}$ in terms of $E$, we find from Eqs. (41), (42), (49), (50), and Poisson's equation, the dispersion relation,

$$
\begin{aligned}
\epsilon(k, \omega) & =1+\chi_{e}+\chi_{l}+\chi_{h} \\
\chi_{e} & =\left(k \lambda_{d e}\right)^{-2} \\
\chi_{h} & =-\frac{\omega_{p h h}^{2}}{\omega(\omega+i \widehat{\nu})}\left(1-\frac{Z_{l} N_{l}}{Z_{h} N_{h}} \eta J_{4}\right)^{2}, \\
\chi_{l} & =\frac{\omega_{p l l}^{2}}{k^{2} V_{l}^{2}} J_{7}
\end{aligned}
$$


where $V_{l, h}$ is the light,heavy ion thermal thermal velocity. The effective damping rate,

$$
\widehat{\nu}=\frac{m_{l} N_{l}}{m_{h} N_{h}} \nu_{1}^{t h}\left(\frac{1}{3} \sqrt{\frac{2}{\pi}+\eta} J_{1}\right)
$$

where $\nu_{1}^{t h}=\nu_{1}\left(V_{l}\right)$ and where $\eta=i\left(\omega / k V_{l}\right) / k \lambda_{l h}$. [18] The kinetic properties are contained in the dispersion functions, $J_{m}(k, \omega)$, defined by

$$
J_{m}=\sqrt{\frac{2}{\pi}} \int_{0}^{\infty} d V \frac{V^{m} \exp \left(-V^{2} / 2\right)}{V^{5}-3 \eta\left(1-i \omega / \nu_{1}\right) H_{1}}
$$

where the dimensionless function, $\left.H_{1}(k, \omega, v)=\nu_{1}^{*}(k, \omega, v) /\left(\nu_{1}(v)-i \omega\right)\right)$, has been introduced.[13] In the next section, we consider examples of the frequency and damping of slow and fast acoustic waves by solving Eq. (52) for complex $\omega$ for a given $k$.

\section{FREQUENCY AND DAMPING OF ACOUSTIC WAVES FOR HEAVY AND LIGHT ION PLASMAS}

In the hydrodynamic equations of Sec II A, the dissipative mechanisms were interspecies drag and thermal conduction by the light ions. Consider first the fast wave. If the charge to mass ratio of the two species are equal, the oscillatory velocity of the heavy ion, $U_{h}$, in the electric field will equal that of the light ion, $U_{l}$, and (aside from ion pressure contributions to the flow) the drag term will be zero. On the other hand, the slow mode oscillatory velocities for each species are nearly 180 degrees out of phase and the mode is strongly damped in this fluid limit, $k \lambda_{l h} \ll 1$. For large $k \lambda_{l h}$, collisionless Landau damping will damp the fast wave more and more strongly as the phase velocity of the wave approaches the light ion thermal velocity. The slow mode phase velocity is close to the light ion thermal velocity and is also strongly Landau damped in the collisionless limit. A collisionless example is shown in Fig. 2.

Now we consider the general case where both Landau damping and collisional damping are included by solving Eq. 52 for the slow and fast waves. In evaluating the functions $J_{m}(k, \omega)$, the poles of the integrand must be considered as the Fourier-Laplace transform assumed that the poles were in the upper-half complex-omega plane. The Landau prescription for treating poles below the real axis for the integrand of the plasma dispersion function[16] in Eq. (34) is applied to Eq. (57). For the fast wave, the simple Landau pole that occurs in Eq. (34) is replaced by an increasing number of poles below which the velocity contour must 

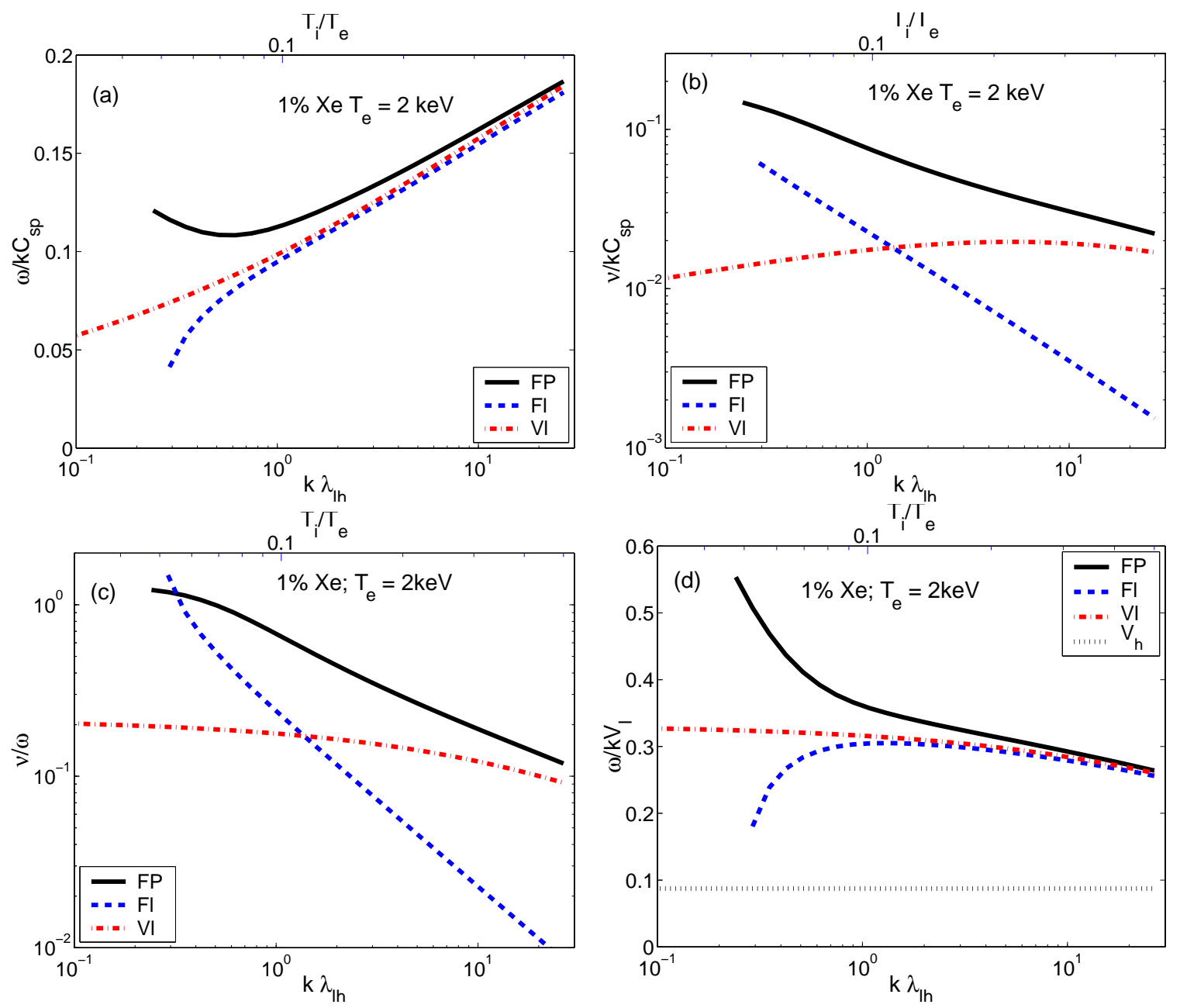

FIG. 3: The frequencies (a) and damping rates (b) normalized to the proton acoustic frequency $k C_{s p}$, the ratios of the damping rate to the frequency (c), and the phase velocities (d) of the slow acoustic waves for the Fokker-Planck Eq. (52) (FP), the fluid (Fl) and the collisionless (Vl) dispersion equations are shown as a function of $k \lambda_{l h}$. The top horizontal axis shows the value of $T_{i} / T_{e}$ for the values of $k \lambda_{l h}$ displayed on the bottom horizontal axis. In (d), the horizontal line, as in Fig. 1, is the xenon $\left(V_{h}\right)$ thermal velocity (normalized to the hydrogen thermal velocity).

be deformed. For the slow wave $\left(\omega / k V_{l}<1\right)$, we do not have to deform the contour below the real axis, and, for that reason, we discuss this computationally simpler case first. 


\section{A. The slow acoustic wave}

Consider again the parameters used in Figs. 1 and 2. For the fluid dispersion, no slow wave roots were found for $k \lambda_{l h}<0.1$. For $0.1<k \lambda_{l h}<20$, collisions are strong at the lower end of the ion temperature range and weak at the high end. The frequency and damping for the Fokker-Planck (FP) dispersion are shown in Fig. 3 along with the fluid (Fl) and Vlasov (Vl) ones. For large $k \lambda_{l h}$, the frequency, damping, and phase velocity approach the collisionless values as expected. However, for small $T_{i}<0.1$ where $k \lambda_{l h} \sim 1$, the frequency departs markedly from the fluid dispersion and, rather than decreasing to zero as the fluid frequency root does, it increases significantly and is larger than the collisionless value. The damping is larger than either the collisional or collisionless value as both Landau damping and collisional damping contribute. The damping becomes as large as the real frequency and, in the intermediate region, it is at least as large as the sum of the damping rates. [There is in both this kinetic dispersion and in the fluid dispersion a zero-frequency, weakly-damped mode for all $T_{i}$ that is distinct from the roots shown in Fig. 3.]

If one assumes a value for $-i \mathrm{kE} / \mathrm{T}_{\mathrm{e}}$, the complex flow velocity for each species can be obtained. Assuming $\psi_{0}=e \phi / T_{i}$ is given where $E=-i k \phi$, one can find the flow velocities,

$$
U_{l, h}=-\frac{\omega}{k} Z_{l, h} \chi_{l, h} k^{2} \lambda_{D l, h}^{2} \psi_{0}
$$

where $\lambda_{D l}\left(\lambda_{D h}\right)$ is the light (heavy) ion Debye length. In Fig. 4, the slow wave flow velocity for the light and heavy (99\% hydrogen and 1\% xenon) ions is shown. The magnitude of the xenon flow is larger than and nearly 180 degrees out of phase (a signature of the slow mode) with that of the hydrogen flow which leads to a large friction force at small $k \lambda_{l h}$.

The behavior of the slow wave for other atomic fractions of $X e$ is similar. If the fraction of $X e$ is increased above $2.5 \%$, most of electrons are supplied by $X e$ and the heavy species dominates the ion susceptability. Nonetheless, the heat flow, friction force, and Landau damping are determined by the light ions. In Fig. 5, the frequency and damping of slow ion acoustic waves are shown from the collisional to collisionless range for $2 \% X e$ and $10 \% X e$. The electron temperature and density are the same as for the $1 \% X e$ examples. Note that the increase in the density of xenon ions reduces the mean free path at a given ion temperature. For $T_{i}=1 \mathrm{keV}$ and constant electron density, $k \lambda_{l h}$ decreases from 26 for $1 \%$ to 17 for $2 \%$

to 10 for $10 \%$ xenon. As is true for $1 \%$ xenon the slow roots are well approximated by 


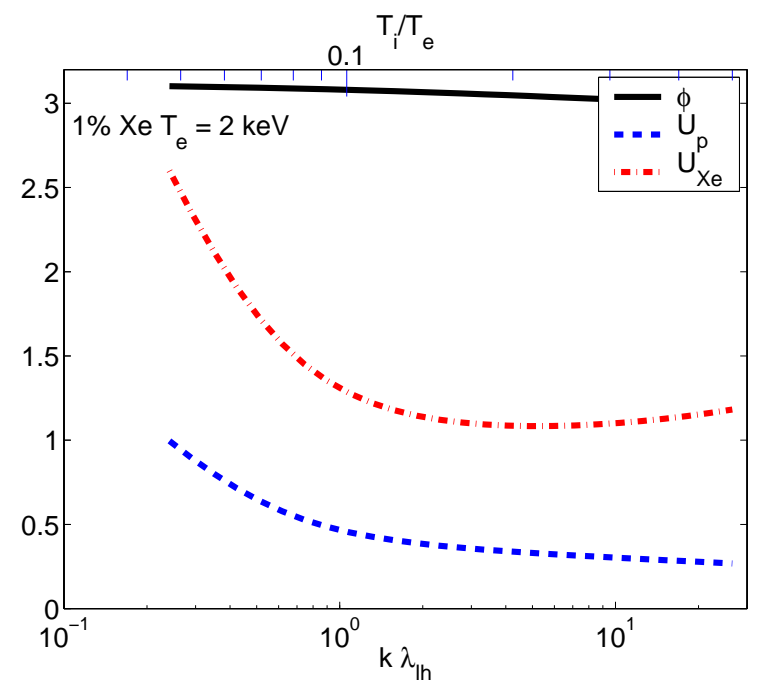

FIG. 4: The magnitude of the hydrogen flow velocity, $U_{p}$, and the xenon flow velocity, $U_{X e}$, for $99 \%$ hydrogen and $1 \%$ xenon for the slow wave. The solid curve shows the relative phase, $\phi_{u}=\cos ^{-1}\left(\mathrm{U}_{\mathrm{Xe}} /\left|\mathrm{U}_{\mathrm{Xe}}\right| / \mathrm{U}_{\mathrm{H}} /\left|\mathrm{U}_{\mathrm{H}}\right|\right)$

the collisionless roots for $k \lambda_{l h}>10$. The real frequency increases with the fraction of $X e$ because $\omega \sim \sqrt{Z_{h}^{2} N_{h}} \rightarrow \sqrt{Z_{h} N_{e}}$. As a consequence, the $10 \% X e$ slow wave phase velocity just exceeds the thermal velocity for small $T_{i}$ for which $k \lambda_{l h}<0.5$. Close examination of these three cases shows that the frequency and damping approach the collisionless values at smaller $k \lambda_{l h}$ for larger $X e$ fraction.

In the case of electron damping of single-species ion acoustic waves, Epperlein[13] showed that the total damping was well approximated by the sum of the Landau damping and the collisional damping if the collisional disruption of the Landau damping was accounted for. Such a separation of effects is possible because different groups of electrons are responsible for each. We repeat this analysis for the $1 \% \mathrm{Xe}$ and $10 \% \mathrm{Xe}$ cases and show the results in Fig. 6.

The collisional contributions for $k \lambda_{l h}<1$ are assumed to result from keeping only Eqs. (36) and (37) for $f_{l, 0}$ and $f_{l, 1}$ respectively with $\omega$ neglected in Eq. (37) because $\omega<k V_{l}<\nu_{l h}$. This collisional diffusive approximation replaces the denominator in Eq. (57) with $V^{5}-3 \eta$ and gives the $(\mathrm{Bl})$ curve in Fig. (6). The Landau damping as affected by collisions but without the collisional damping from $R_{l h}$ is found by setting to zero the RHS of Eq. (37) but retaining the equations $l>1$. In this case the denominator in Eq. (57) becomes 

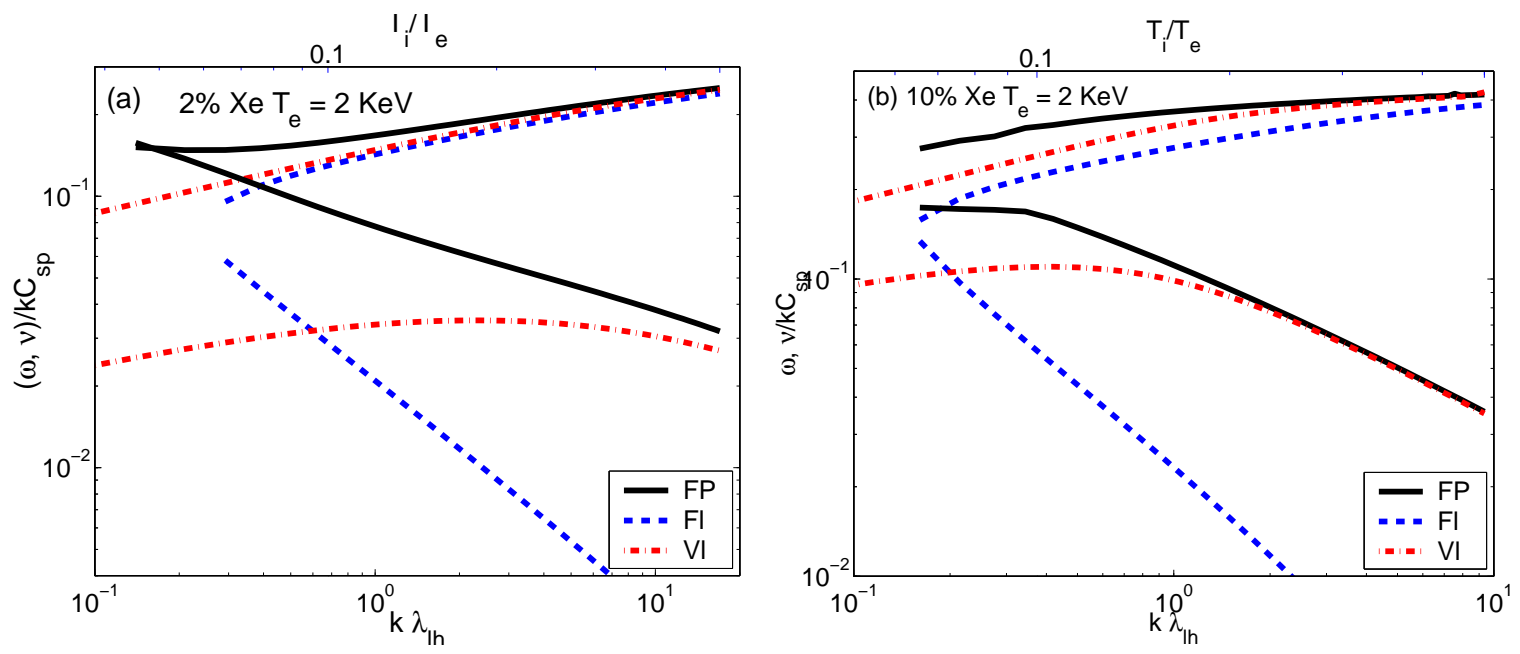

FIG. 5: The frequencies (upper set of curves) and damping rates (lower set) normalized to the proton acoustic frequency $k C_{s p}$ are shown for $2 \% X e$ (a) and $10 \% X e$ (b) for the parameters of Figs. 1 and 2. The curves (FP) are the solutions to Eq. (52), the curves (Vl) are the collisionless roots, and the curves $(\mathrm{Fl})$ are the fluid roots.

$V^{5}-3 \eta\left(\left(1-i \omega / \nu_{1}\right) H_{1}-1\right)$ and the terms proportional to $U_{l, h}$ in the equations (46) and (48) for $f_{l, 0}$ and $f_{l, 1}$ respectively do not appear. That approximation gives the (Ld) curve. The Landau damping is strongly reduced for small $k \lambda_{l h}$ with the onset of the reduction at larger $k \lambda_{l h}$ for the plasma with a smaller fraction of xenon. This behavior is consistent with the fact that the FP roots approach the collisionless roots at smaller $k \lambda_{l h}$ for larger fractions of xenon. The sum of the damping rates, (legend:sum), falls below the damping for the full dispersion for the intermediate range of collisionality, which is $0.1<k \lambda_{l h}<1$ for $10 \%$ xenon and $1<k \lambda_{l h}<10$ for $1 \%$ xenon. On the other hand, the sum of the damping rates for the two approximations overstates slightly the damping in the collisionless limit, $k \lambda_{l h} \gg 1$, as is apparent for the $10 \%$ Xe case. The separation of effects of friction, ion heat flow, and Landau damping does not work as well for ion-ion collisions as for electron-ion collisions because of the ratio of the ion mass is not large enough to separate the regions of velocity space that contribute to each process. The light ions that Landau damp the wave have velocities, $v \sim \omega / k$, that are not much different from the velocities of the ions that collisionally damp the wave, $v \sim\left(3(\omega / k) /\left(k \lambda_{l h}\right)\right)^{1 / 5}$.

The real part of the frequency for the collisionally reduced Landau damping roots follows the collisionless dispersion even as the Landau damping decreases and the collision rate $\nu_{l h}$ 

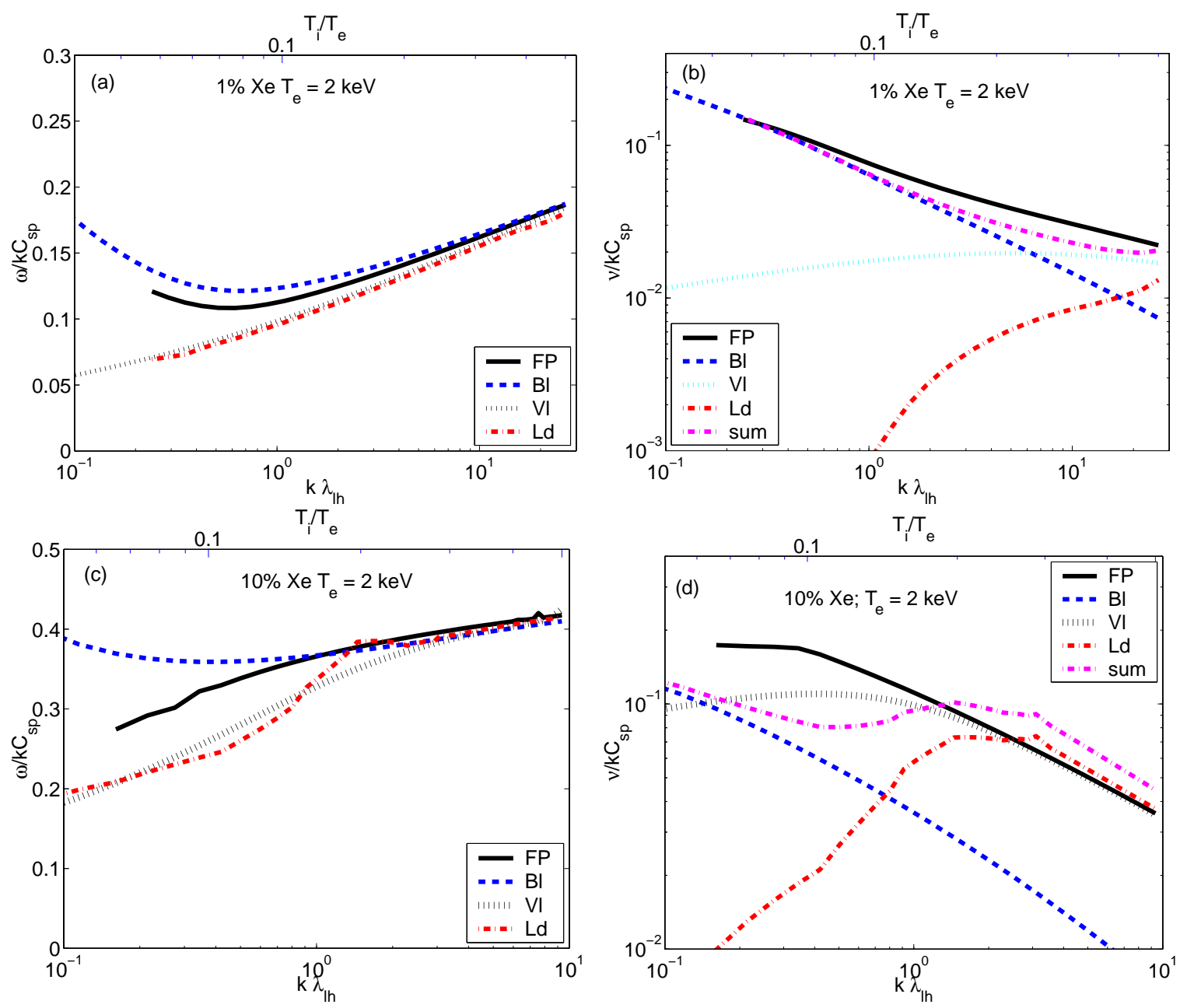

FIG. 6: The slow wave frequency (a and c) and damping (b and d) divided by $k C_{s p}$ for a $1 \%$ xenon $99 \%$ hydrogen plasma (a and b) and for a 10\% xenon $90 \%$ hydrogen plasma (c and d) as a function of $k \lambda_{l h}$. The curves (FP) are the solutions to Eq. (52); the curves (Ld) are collisionally disrupted Landau roots found by setting to zero the RHS of Eq. (37); the curves (Bl) are the diffusion approximation roots keeping only Eqs. (36) and (37) with $\omega$ neglected in Eq. (37). The sum of the damping from the collisionally disrupted Landau and the diffusion approximation is also shown (legend:sum) . The curves ( $\mathrm{Vl}$ ) show frequency and damping from the collisionless dispersion.

increases. For the slow modes, the phase velocity is nearly a constant fraction of the light ion thermal velocity such that $\omega / k V_{l}=0.3$ for $1 \%$ xenon and $\omega / k V_{l} \sim 1$ for $10 \%$ xenon. Thus the criterion that the collisional effects become apparent once $\nu_{l h} \gtrsim|\omega|$ explains the different $k \lambda_{l h}$ at which the onset of collisional effects occurs for the $1 \%$ and $10 \mid \%$ cases. 


\section{B. The fast acoustic wave}

The fast wave frequency is proportional to $\sqrt{T_{e}}$ for $T_{i} / T_{e}<<1$. Thus Landau damping is very small for small $T_{i} / T_{e}$ and becomes increasing important as the ion temperature increases as shown in Fig. 2. On the other hand, collisional damping becomes important for small $k \lambda_{l h}$ as shown in Fig. 1. Finding fast wave roots of the full dispersion relation requires evaluation of the integral in Eq. (57). For small $k \lambda_{l h}$, examination of the integrand in Eq. (57) shows no poles that cross the real velocity axis as the imaginary part of $\omega$ moves from positive values to the negative value corresponding to a root of the dispersion relation. However, as $k \lambda_{l h}$ increases, first one, then many such poles do appear. For $k \lambda_{l h} \sim 10$ and the parameters of Fig. 1, there are four such poles for the complex frequency that is a fast wave root of the collisionless dispersion relation. Using that frequency and its poles as an initial guess, one finds roots very close to the initial guess and no other roots with smaller damping. In Appendix B, an example of the behavior of the denominator of the generalized dispersion integrals is shown. Following this procedure for smaller $T_{i}$, one gets roots with real part near the Vlasov root but with more damping than the collisionless Landau damping until no poles below the real axis are found. That crossover point occurs just where the fluid theory roots have more damping than the Vlasov roots, i.e., $T_{i}<0.1 \mathrm{keV}$ and $k \lambda_{l h} \sim 1$. In Fig. 7, the frequencies, damping rates, and phase velocities of the fast wave for $1 \%$ xenon are shown for the full dispersion (FP), the fluid dispersion (Fl), and the collisionless dispersion (Vl). The frequency from the full dispersion agrees with the fluid dispersion in the collisional limit and with the Vlasov dispersion in the collisionless limit. The full dispersion damping rate also agrees with the damping rates from the fluid and Vlasov dispersion in their regimes of validity. There is only a small region of $k \lambda_{l h}$ where one of the limits does not work well.

As we did for the slow wave roots, we isolate the effect of the collisions on the Landau damping by setting the friction force to zero by neglecting the explicit collisional term (the RHS of Eq. (37) ) in the equation for $\delta f_{l, 1}$. [There is no fast wave solution if $\omega=0$ in Eq. (37) and the $l>1$ contributions are neglected; thus, there is no analogue to the diffusive approximation used for the slow wave when $k \lambda_{l h}<1$.] That root's dependence on $k \lambda_{l h}$ is also shown in Fig. 7. The frequency, damping, and phase velocity are the same as the collisionless dispersion at short wavelengths. At long wavelengths, the frequency lies between the fluid (Fl) (and FP) root and the collisionless root (Vl). As a result, collisions 

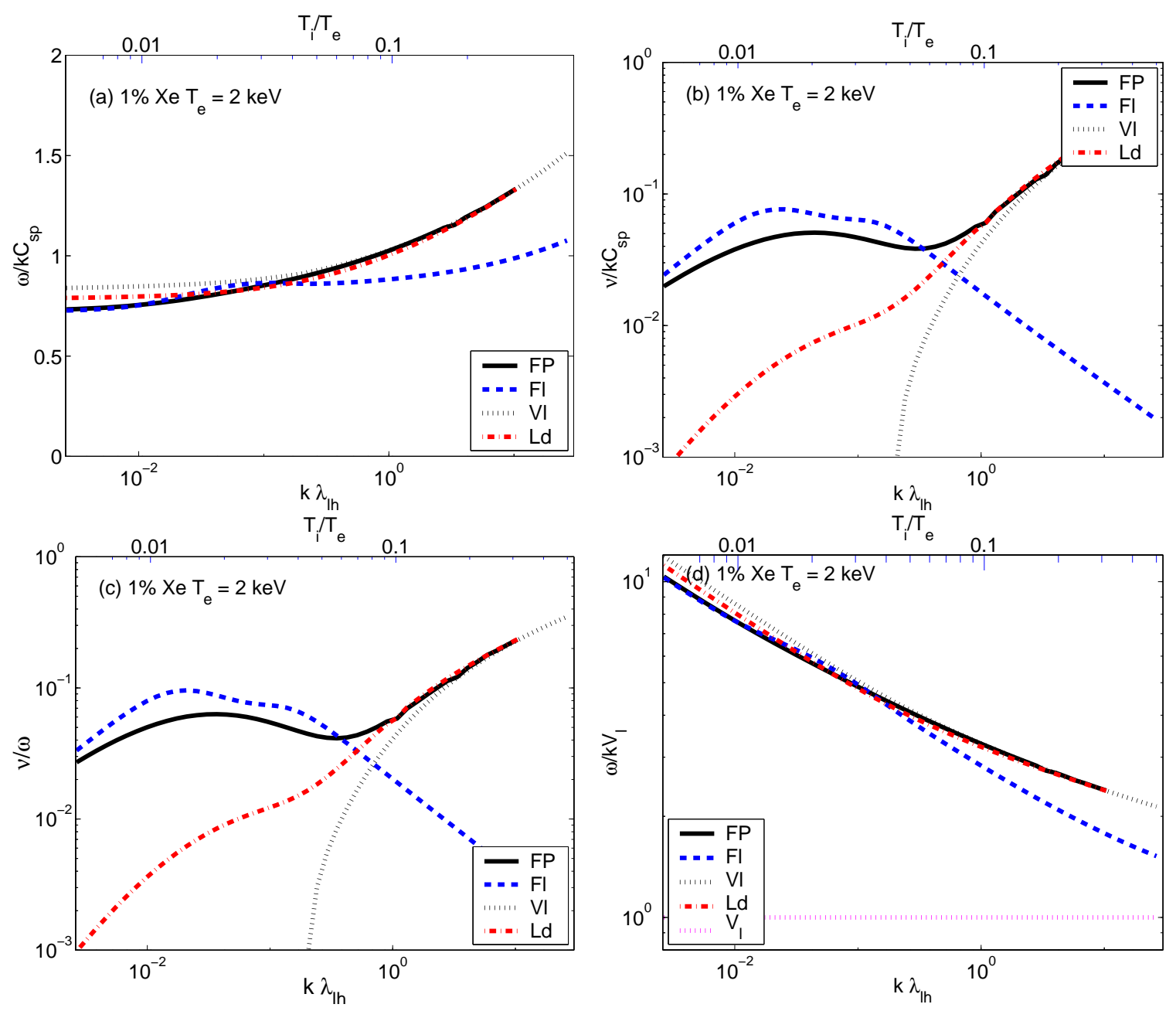

FIG. 7: The frequencies (a) and damping rates (b) normalized to the proton acoustic frequency $k C_{s p}$, the ratios of the damping rate to the frequency (c), and the phase velocities (d) of the fast acoustic waves for the Fokker-Planck Eq. (52)(FP), the fluid (Fl) and the collisionless (Vl) dispersion equations are shown as a function of $k \lambda_{l h}$ for the parameters of Figs. (1) and (2). Also shown is the collisional disrupted Landau dispersion (Ld). The top horizontal axis shows the value of $T_{i} / T_{e}$ for the values of $k \lambda_{l h}$ displayed on the bottom horizontal axis. In (d) the light ion thermal velocity, $\left(V_{l}=1\right)$, is also shown.

(in this artificial case) increase the ion Landau damping rather than decrease it, although not enough to make ion Landau damping important.

If one assumes a value for $-i \mathrm{kE} / \mathrm{T}_{\mathrm{e}}$, the complex flow velocity for each species can be obtained from Eq. (58). In Fig. 8, the fast wave flow velocity for the light and heavy (99\% 


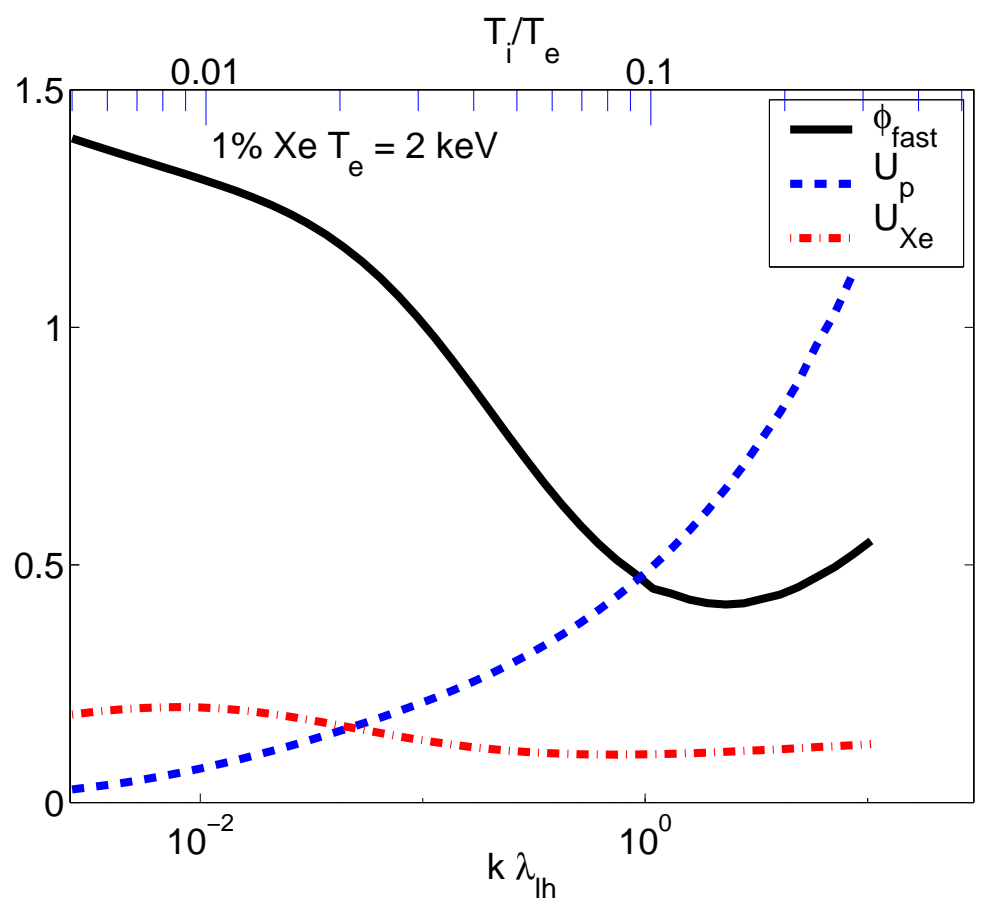

FIG. 8: The magnitude of the hydrogen flow velocity, $U_{p}$, and the xenon flow velocity, $U_{X e}$, for $99 \%$ hydrogen and $1 \%$ xenon for the fast wave. The $\left(\phi_{f a s t}\right)$ curve shows the relative phase, $\phi_{\text {fast }}=\cos ^{-1}\left(\mathrm{U}_{\mathrm{Xe}} /\left|\mathrm{U}_{\mathrm{Xe}}\right| / \mathrm{U}_{\mathrm{H}} /\left|\mathrm{U}_{\mathrm{H}}\right|\right)$

hydrogen and $1 \%$ xenon) ions is shown. In contrast with the slow wave, here as expected, the hydrogen flow has a magnitude larger than the xenon flow velocity in the collisionless limit. In this limit, the two species oscillate nearly in phase with a relative phase of about 30 degrees. In the collisional regime, the magnitudes of the flow velocities, $\left|U_{X e}\right|$ and $\left|U_{H}\right|$, are small with relative phase of 90 degrees. Thus, despite the large collision rate, $\nu_{l h}$, the friction force plays less of a role for fast waves than for slow waves both because the amplitude of the flows are about $10 \times$ less than for the slow wave in the collisional regime and because the phase difference is smaller.

The behavior of the fast wave roots as the ion temperature or light ion mean free path is varied for $2 \%$ and $10 \% \mathrm{Xe}$ is similar to the $1 \%$ case as is shown in Fig. 9. Again, the frequency and damping rate for the full (FP) dispersion agrees with the fluid (Fl) dispersion at long wavelengths and the collisionless (Vl) dispersion at short wavelengths. The damping rate for the full (FP) dispersion is about the same as the sum of the Landau (Vl) and collisional (Fl) damping. The value of $k \lambda_{l h}$ for which the transition from collisional to collisionless 

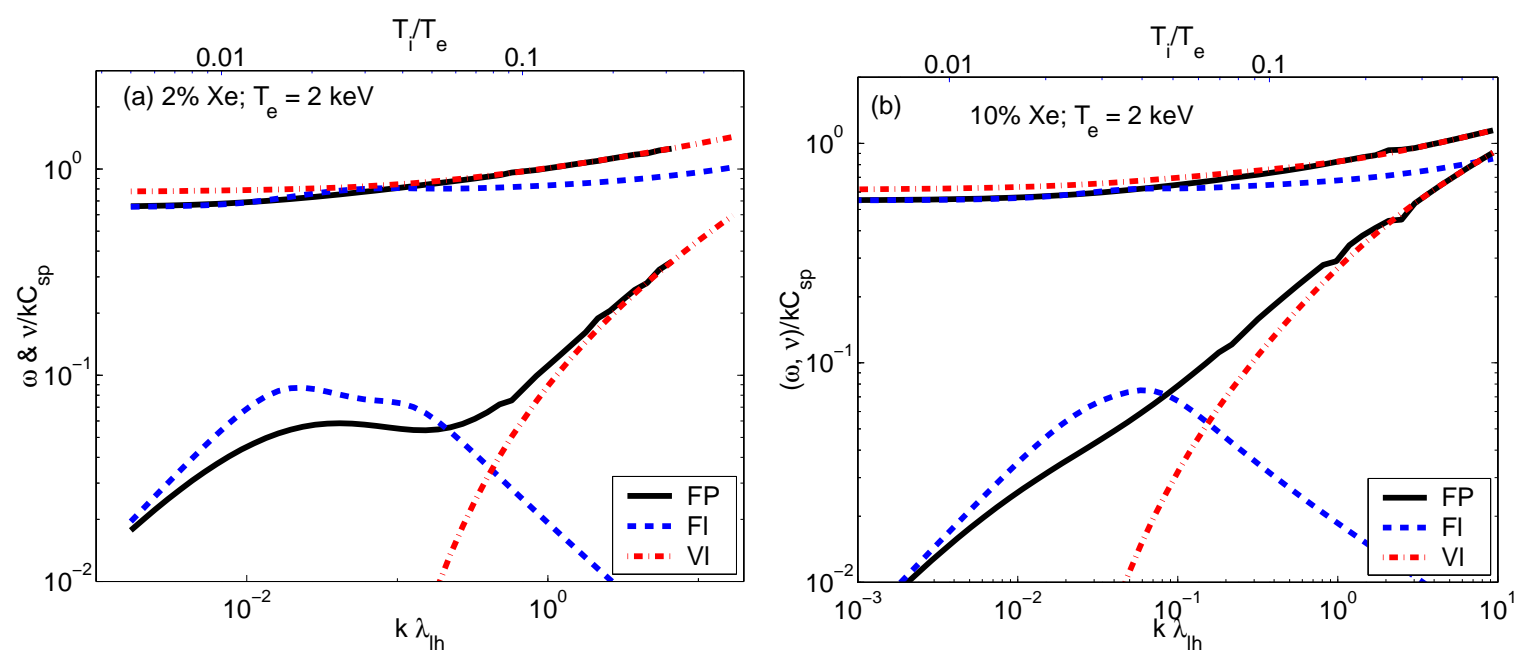

FIG. 9: The fast wave frequency and damping rate normalized to the proton acoustic frequency $k C_{s p}$ for $2 \% X e(\mathrm{a})$ and $10 \% X e(\mathrm{~b})$ for the parameters of Figs. 1 and 2 . The (FP) curves are the solutions to Eq. (52), the (Vl) curves are the collisionless roots, and the (Fl) curves are the fluid roots. The top horizontal axis shows the value of $T_{i} / T_{e}$ for the values of $k \lambda_{l h}$ displayed on the bottom horizontal axis.

behavior occurs $\left(\omega \sim \nu_{l h}\right)$ decreases slightly as the fraction of xenon increases because the frequency of the fast wave decreases with increasing fraction of xenon.

\section{Behavior versus wavenumber for constant $T_{i}$}

The behavior of the roots as $k \lambda_{l h}$ varied was studied in the previous examples by variation of the ion temperature for a fixed acoustic wave wavelength. Now, consider the variation of $k \lambda_{l h}$ and its effect on the acoustic wave as the wavenumber is varied for fixed $\mathrm{T}_{\mathrm{i}}$. Long wavelength modes will be affected more by collisions than short wavelength modes. In Figs. 10, the roots of the full dispersion for $k \lambda_{l h}=1.5$ for which $\mathrm{T}_{\mathrm{i}}=0.4$ and $\mathrm{k} / \mathrm{k}_{0}=2$ $\left(k_{0}=2 \pi / \lambda_{0}\right.$ and $\left.\lambda_{0}=351 \mathrm{~nm}\right)$ agree with the collisionless dispersion. In the collisionless limit, varying $k$ has little effect on the dispersion, $(\omega, \gamma \simeq k)$, unless $k \sim \lambda_{d e}^{-1}$. Similarly the real frequency that satisfies the fluid dispersion is linearly proportional to $k$, albeit with a different constant of proportionality, except at very small $k \lambda_{l h}$. The damping of the fast and slow waves according to the fluid dispersion behaves as shown in Figs. 10 for $k \lambda_{l h}<1.5$. The slow wave roots to the dispersion that includes both collisions and Landau damping 

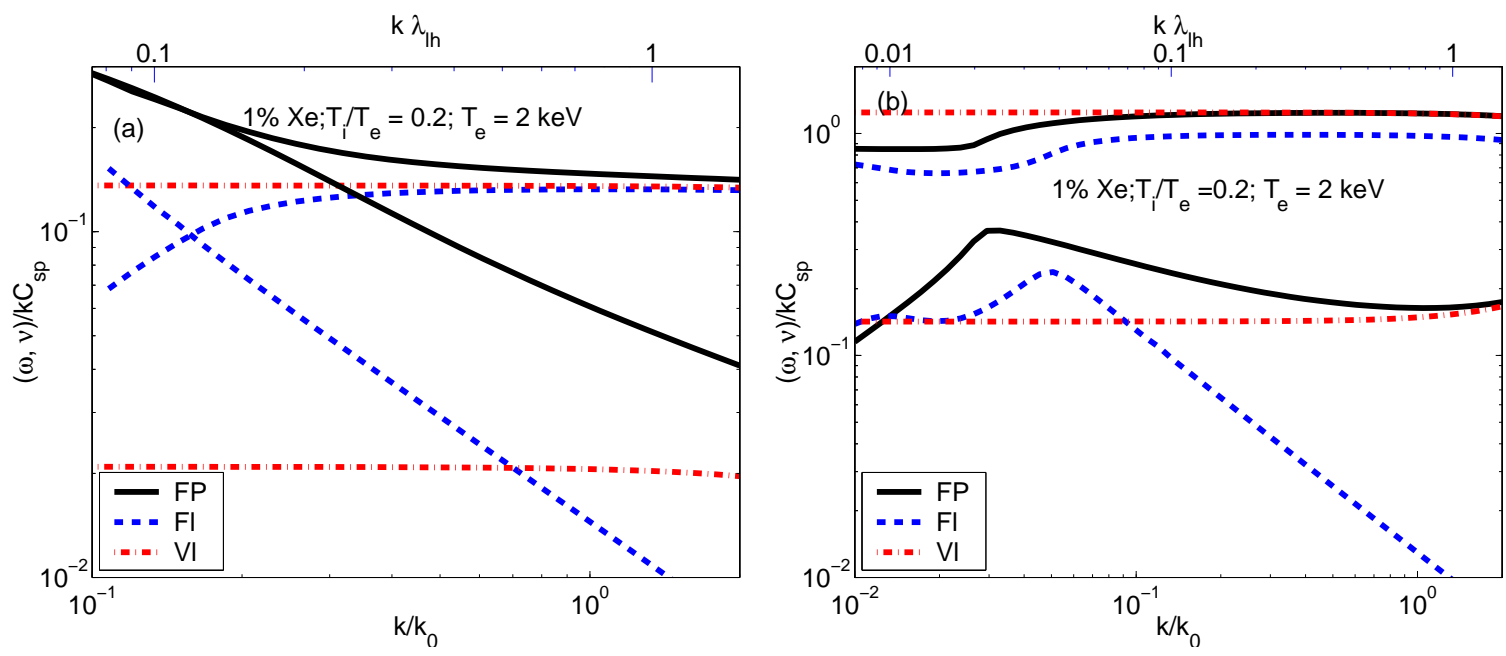

FIG. 10: The slow wave (a) and fast wave (b) frequency and damping rate normalized to the proton acoustic frequency $k C_{s p}$ are shown as a function of wavenumber $k / k_{0}$ where $k_{0}=2 \pi / \lambda_{0}$, $\lambda_{0}=351 \mathrm{~nm}, T_{i}=0.4 \mathrm{keV}$, and $1 \%$ Xe by atom. The (FP) curves are the solutions to Eq. (52), the (Vl) curves are the collisionless roots, and the $(\mathrm{Fl})$ curves are the fluid roots. The top horizontal axis shows the value $k \lambda_{l h}$ corresponding to the $k / k_{0}$ displayed on the bottom horizontal axis.

have a complex phase velocity $\omega / k$ and $\gamma / k$ that increase as the wavenumber decreases until the $\gamma \sim \omega$. This is also the point where the fluid dispersion no longer supports slow wave roots.

The behavior of the fast wave as $k$ is varied is also shown in Fig. 10. Here, for $k / k_{0}>0.1$, the phase velocity follows the collisionless dispersion, that is $\Re(\omega) / k=$ constant. However, the damping, $\gamma / k$, increases from collisional processes as $k$ is decreased. Once $k \lambda_{l h} \cong 0.2$ $\left(k / k_{0} \cong 0.1\right) \gamma / \omega$ increases and $\omega(k)$ departs markedly from the collisionless dispersion as $k / k_{0}$ decreases. The frequency decreases as $k$ decreases for $k / k_{0}<0.1$ as does the fluid dispersion relation for this range of parameters until a maximum $\gamma / \omega$ is reached for very small wavenumbers. Note, over the range $k / k_{0}<.03$, there are no poles below the real velocity axis contributing to the integrand for Eq. (57).

This dependence of the damping on $k \lambda_{l h}$ is potentially important for propagation of light through large regions of underdense plasma because the amplification of stimulated forward Brillouin scatter is proportional to $\omega / \nu$ when the growth rate is small, i.e. $\gamma_{s b s}<\nu$. To our knowledge, simulations of light propagation treat the ion waves with an arbitrary viscosity coefficient or a Landau-like term such that $\nu / \omega$ is constant. 

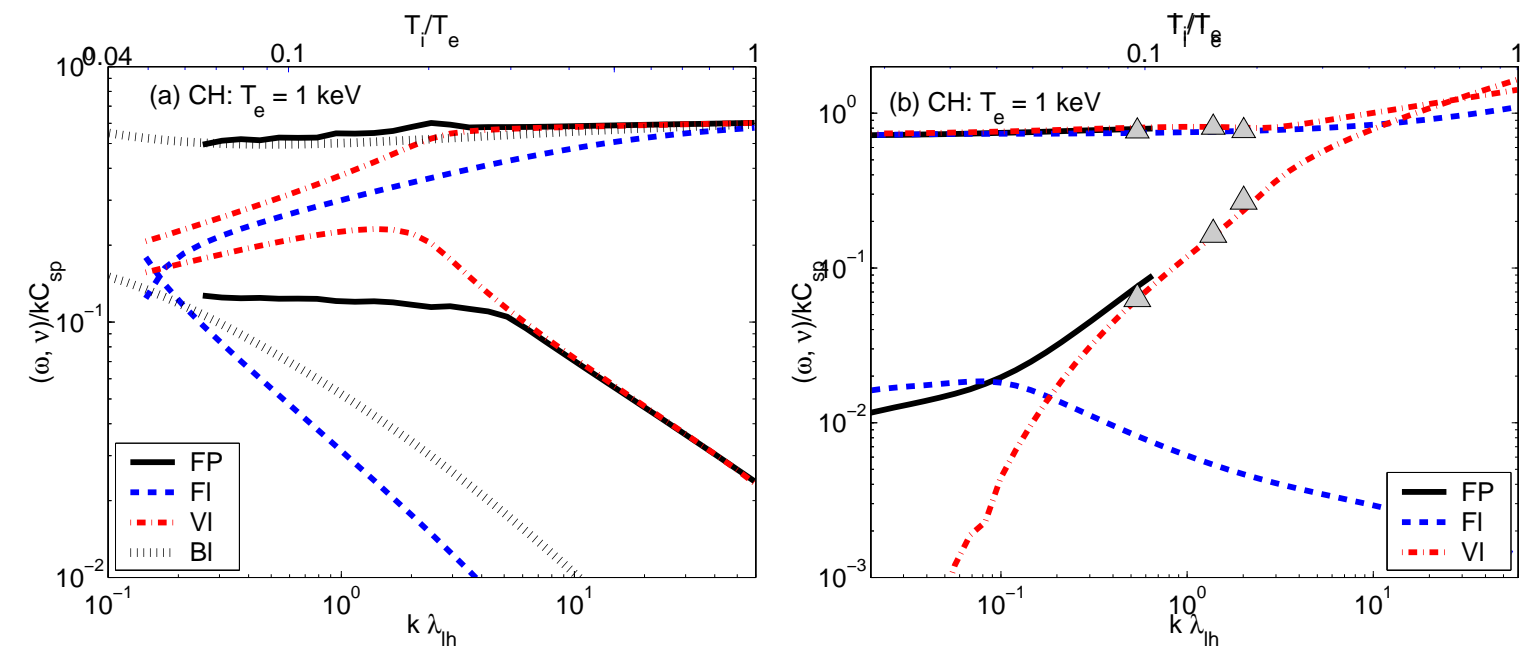

FIG. 11: The slow (a) and fast (b) wave frequency and damping rate normalized to the proton acoustic frequency $k C_{s p}$ for a $\mathrm{CH}$ plasma. The (FP) curves are the solutions to Eq. (52), the (Vl) curves are the collisionless roots, the $(\mathrm{Fl})$ curves are the fluid roots, and the $(\mathrm{Bl})$ curves are the roots in the diffusive limit where only $\delta f_{0}$ and $\delta f_{1}$ are kept. For $T_{i}>0.1$, the full dispersion fast wave roots, found only for a few values of ion temperature, are plotted as solid $\triangle \mathrm{s}$. The top horizontal axis shows the value of $T_{i} / T_{e}$ for the values of $k \lambda_{l h}$ displayed on the bottom horizontal axis.

\section{Mixtures of hydrogen and carbon}

The discussion to this point has been limited to mixtures of partially ionized high $\mathrm{Z}$ and ionized hydrogen gas because the neglect of ion self-collisions and of kinetic effects in the heavy ions is clearly justified. In addition, the fast and slow wave mode frequencies are well separated. Here we show that the influence of ion-ion collisions are also important in the damping of ion acoustic waves in hydrocarbon plasmas, the most often-used plasma for studying laser-plasma interactions. For example, consider a plasma of equal number of protons and fully ionized carbon atoms at an ion temperature of $0.1 \mathrm{keV}$ and an electron density of $1 \times 10^{21} \mathrm{~cm}^{-3}$, a typical value for Inertial Confinement Experiments. For the ion wave wavenumber that Brillouin backscatters a $351 \mathrm{~nm}$ wavelength light wave, $k \lambda_{l h}=0.6$, a value that is neither clearly collisional nor clearly collisionless. Further, taking an electron temperature of $1 \mathrm{keV}$ also a typical value, we find the fast wave dispersion is slightly but the slow wave is substantially altered. The behavior of the fast and slow wave modes of a 
$T_{e}=1 \mathrm{keV}$, fully-ionized $C H$ plasma is shown in Fig. 11 as a function of ion temperature from $.04 \mathrm{keV}$ to $1 \mathrm{keV}$ for which $k \lambda_{l h}$ varies from 0.006 to 60 . The longer wavelength acoustic waves that are involved in Brillouin forward scatter are strongly affected by ion-ion collisions. The slow wave frequency and damping are markedly different from either approximate (fluid or collisionless) solution for $T_{i}<0.2 \mathrm{keV}$. It is interesting to find the modes in the diffusive $(\mathrm{Bl})$ approximation where only $\delta f_{0}$ and $\delta f_{1}$ are retained such that the Landau resonance is eliminated. That is also shown in Fig. 11a. The frequency for this (Bl) case follows that of the FP frequency but the damping is much reduced. Thus, the slow wave damping for $k \lambda_{l h} \lesssim 3$ is mostly Landau damping but with a frequency strongly influenced by collisions. The (FP) Landau damping is less than the collisionless theory (curve Vl) predicts because the phase velocity is greater than collisionless theory predicts. In fact, its phase velocity for $T_{i}<0.2 \mathrm{keV}$, while less than that of the fast wave, is no longer less than the light ion thermal velocity. Following the slow wave roots in the diffusive limit to $k \lambda_{l h}=.01$, one finds that they merge with the fast wave roots.

The fast wave shown in Fig. $11 \mathrm{~b}$ is still the least damped wave. For $T_{i}>0.1$, the FokkerPlanck fast wave roots are essentially the same as the collisionless roots. For $T_{i} / T_{e}<0.1$, the fast wave ion damping, because of ion-ion collisions, is slightly larger than the electron Landau damping value that is typically assumed.

\section{The perturbed distribution function}

The perturbation to the distribution function $\delta f_{l}$ in Eq. (35) that corresponds to a given normal mode frequency can be reconstructed once the complex frequency is obtained and the relation between the flow and the electric field in Eq. (58) is used. Then the lowest order Legendre coefficients are obtained from Eqns. (46) -(48) and the higher order ones from Eqs. (43) and (38). One expects that Landau damping will produce a perturbation localized to velocities whose component along $\mathrm{k}, v_{\|}=\omega / k$ whereas the thermal diffusion will produce features where $v_{d}^{5}=3 i \omega / k V_{l} / k \lambda_{l h}$. The perturbed distribution functions for the various approximations discussed for Fig. 6 are shown for the slow wave in Fig. 12. For the slow wave of a $1 \%$ xenon, $99 \%$ hydrogen plasma, the intermediate region of parameter space where different damping processes are comparable has typical parameters, $T_{i}=0.52, k \lambda_{l h}=7.1$ as is shown by Fig. 6 for which the complex normal mode frequency from the Fokker-Planck 

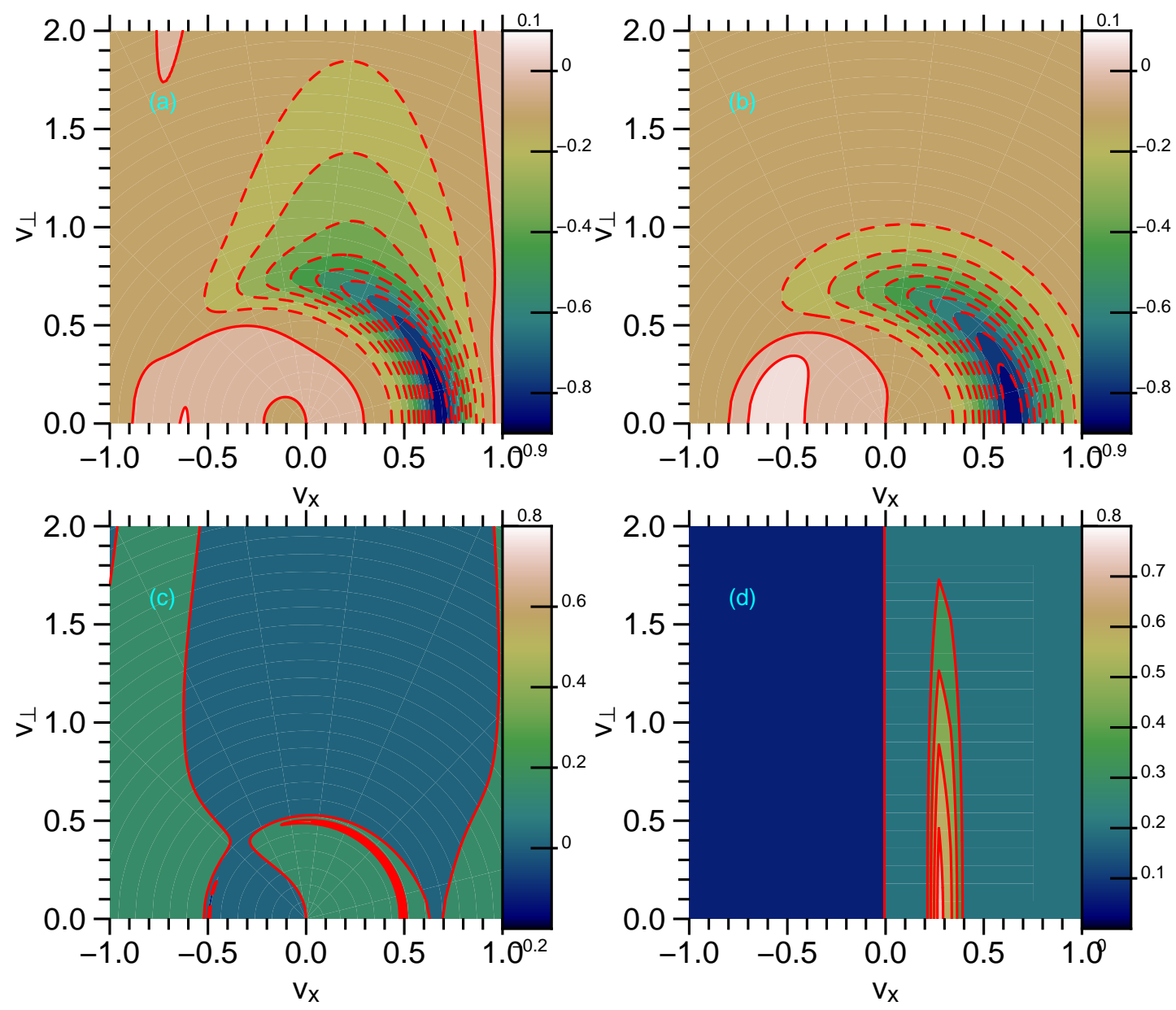

FIG. 12: The imaginary part of the perturbed distribution, $\delta f_{l}\left(v_{\|}, v_{\perp}\right)$, for the slow wave mode with $k \lambda_{l h}=7.1$ is shown for (a) the Fokker-Planck solution, (b) the Fokker-Planck solution in the diffusive limit with the $l>1$ terms omitted, (c) the Fokker-Planck solution with the $l=1$ collision operator omitted, and (d) the Vlasov equation solution. The contour levels for (a) and (b) run from -0.9 to 0.1 in increments of 0.1 . The contour levels for (c) and (d) run from -0.2 to 0.8 in increments of 0.2. Negative contour levels are dashed lines.

(FP) dispersion, $\omega / k V_{l}=0.3-.07 i$. Thus, the Landau resonance is at $v_{\|} / V_{l}=0.3$ and the ions responsible for the diffusive damping have velocities, $v_{d} \sim 0.6 V_{l}$. Both these features are visible in Fig. 12. In the diffusive limit, the Landau resonance feature disappears. The Vlasov distribution is also shown in Fig. 12. In the collisionless limit, the resonant particles occupy a narrower region of velocity space than for the Fokker-Planck distribution. For intermediate values of $v_{\perp} \sim 0.5-1.0 V_{l}$ and $v_{\|}=\omega / k$, these two regions of velocity space overlap. 
For the fast wave, consider the $1 \%$ xenon example with $k \lambda_{l h}=1.6$ for which one expects from Fig. 7 the Landau damping to be the dominant contributor. Then the Landau resonance is at $v_{\|} / V_{l}=3$. and $v_{d}=1.3 V_{l}$. In Fig 13 , the perturbed distribution is shown with the collisions included, for the collisions included only in the equations for $\delta f_{l}$ when $1>1$ and, for reference, the perturbed distribution for the Vlasov equation. Where the Vlasov perturbation shows only a feature at the phase velocity, the Fokker-Planck perturbation shows that feature but also perturbations at velocities $v<2$. These low velocity features, unique to the full dispersion, originate in the collisional interaction as is clear from the distribution in Fig. 13 that is formed without the collision operator in the equation for $\delta f_{l=1}$. The collisional feature moves to higher velocity and becomes more prominent for longer wavelengths. The collision operator works to isotropize the Landau resonance as can be seen by comparing the Vlasov perturbed distribution with the others.

\section{DISCUSSION}

The discussion has centered on the complex roots of the plasma dispersion function with the inclusion of ion-ion collisions. One consequence of an improved theory would be a different interpretation of the Thomson scattering spectrum from plasmas with heavy ions. If the modes of the plasma are weakly damped, the spectrum is proportional to $\Im(1 / \epsilon)$ where $\epsilon(k, \omega)$ is given by Eq. (52). For the hydrogen-xenon plasmas that have been the principal example in this study, there are two distinct peaks in the spectrum that correspond to the slow and fast waves. The ratio of the heights of the peaks depends on the relative damping of the modes; that and the position of the peak can be used to determine the electron and ion temperature if the dispersion relation and wavenumber $k$ are known. In Fig. 14, we consider two examples of Thomson scattering spectra, the $99 \%$ hydrogen and $1 \%$ xenon plasma and the $99 \%$ beryllium and $1 \%$ gold plasma used in an experiment.[2] In both cases, $k=2 k_{0}$ where $k_{0}$ is the wavenumber of a $351 \mathrm{~nm}$ light wave in the plasma. In the hydrogen-xenon example, the fast wave spectral feature (the phase velocity of the fast wave relative to the

proton sound speed, $\sqrt{T_{e} / m_{p}}$ ) is somewhat higher when the collisions are included. The width of the fast wave spectral feature is about the same with and without collisions. The shift of slow wave spectral feature (phase velocity relative to the proton sound speed) is independent of both the ion temperature and the inclusion of collisions. However, the ratio 

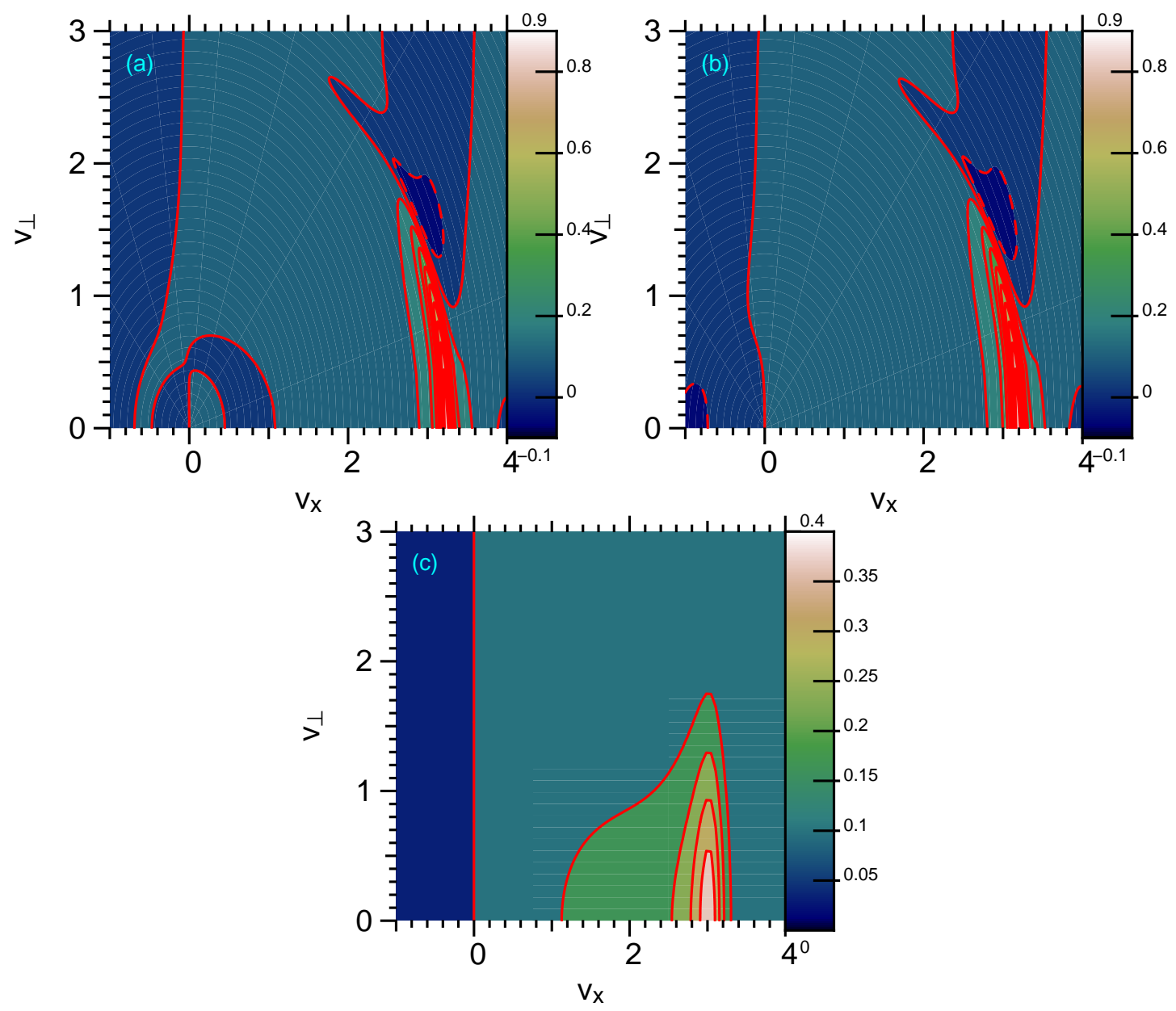

FIG. 13: The imaginary part of the perturbed distribution, $\delta f_{l}\left(v_{\|}, v_{\perp}\right)$, for the fast wave mode with $k \lambda_{l h}=1.6$ is shown for (a) the Fokker-Planck solution, for (b) the Fokker-Planck solution without the collision operator in $\delta f_{l=1}$, and (c) the Vlasov equation solution. For (a) and (b), the contour levels run from -0.1 to 0.9 in increments 0 f 0.1 . For (c) the levels are from 0 . to 0.4 in increments of 0.1 .

of the peaks of the spectral features is sensitive to collisions as the slow wave is more strongly damped when collisions are included. For $T_{i}=500 \mathrm{eV}, k \lambda_{l h}=6$ for the hydrogen-xenon plasma. The gold-beryllium plasma does not have a distinct feature corresponding to the slow wave with or without collisions at either ion temperature considered (100eV and $300 \mathrm{eV})$ The absence of a distinct slow wave feature is consistent with the experimental result [2]. The fast wave feature is, for both ion temperatures, at a higher phase velocity with collisions than without. With and without collisions, the fast wave spectral feature is much narrower at the lower temperature. For $T_{i}=300 \mathrm{eV}, k \lambda_{l h}=4$ for the beryllium-gold plasma. These 

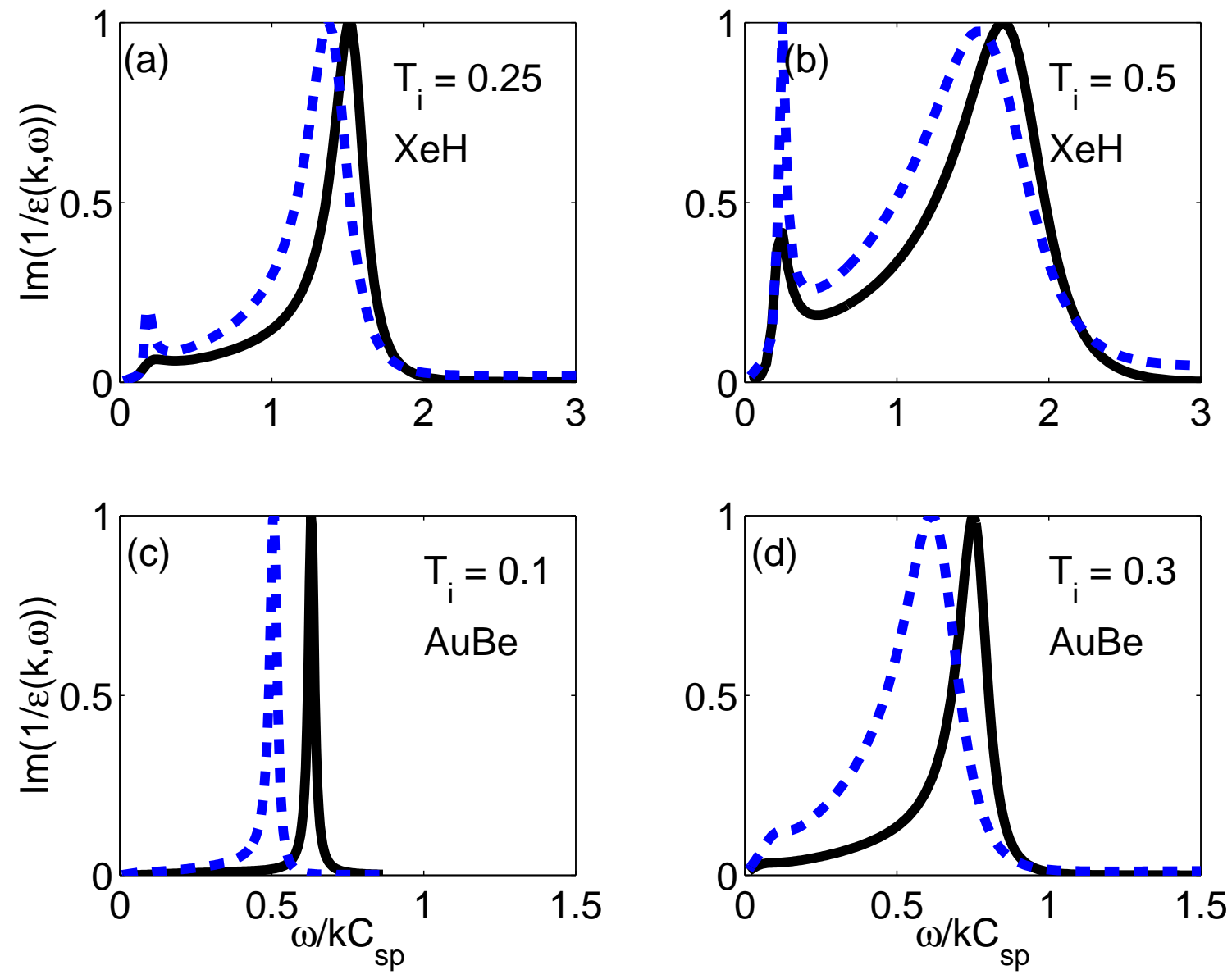

FIG. 14: The Thomson scattering spectrum (a and b) for a xenon-hydrogen (XeH) plasma and (c and d) for a gold-beryllium (AuBe) plasma. The spectra from the Fokker-Planck (solid) and the collisionless (dashed) dispersion are shown. For the XeH plasma, $T_{e}=1 \mathrm{keV}, N_{e}=1 \times 10^{21} \mathrm{~cm}^{-3}$, and the atomic fraction of $\mathrm{Xe}$ is $1 \%$. Spectra for ion temperatures of $250 \mathrm{eV}$ (a) and $500 \mathrm{eV}$ (b) are displayed. For the AuBe plasma, $T_{e}=600 \mathrm{eV}, N_{e}=1 . \times 10^{20} \mathrm{~cm}^{-3}$, and the atomic fraction of $\mathrm{Au}$ is $1 \%$. Spectra for ion temperatures of $100 \mathrm{eV}$ (c) and $300 \mathrm{eV}$ (d) are displayed.

examples show that quantitative conclusions about the electron and ion temperatures may still be made but the values obtained from a collisionless theory may be misleading. 


\section{APPENDIX A: THE DEFINITION OF THE RENORMALIZED COLLISION FREQUENCY}

The effects of the higher order Legendre polynomial coefficients on the lowest order ones, $f_{l, 0}$ and $f_{l, 1}$, which determine the light ion density and the friction force are incorporated by following established procedure.[13] In the equation for the $l^{\text {th }}$ coefficient, define

$$
\nu_{l}^{*} \equiv \nu_{l}-i \omega+i k v \frac{l+1}{2 l+3} \frac{\delta f_{l+1}}{\delta f_{l}}
$$

Then, the equation for the $l^{\text {th }}$ coefficient for $l>2$ coefficient is determined by lower order $l \leq 2$ coefficients provided $\nu_{l}^{*}$ can be found. Using Eq. (A1) recursively, one obtains

$$
\nu_{l}^{*}=\nu_{l}-i \omega+\frac{k^{2} v^{2}}{\nu_{l+1}^{*}} \frac{(l+1)^{2}}{(2 l+1)(2 l+3)},
$$

which is used in Eq. (45). The procedure for solving Eq. (A2) is outlined in Ref. [13].

\section{APPENDIX B: THE INTEGRAND OF THE GENERALIZED DISPERSION FUNCTIONS}

Given a complex $\omega$, the behavior of the real and imaginary part of the integrand in Eq. (57) is found for a range of complex velocities and shown in Fig. 15 for $10 \% X e, k \lambda_{l h}=$ 2.5 , and $\omega / k V_{l}=1.84-0.88 i$. The color represents the value of the real part of the integrand, the lines trace contours where the real (black) and imaginary (red) parts are zero. The different color lines cross where the denominator has either a pole or a zero. The zeros of interest occur in (simple) pole and zero combinations. In this Fig. 15, there are three relevant zeros of the denominator (poles of the integrand) with values $V / V_{l} \sim$ $\left[(2-0.62 i, 2.3-0.27 i, 2.7-0.06]\right.$. The other zeros with $\operatorname{ImV} / V_{l}<-0.62$ remain below the real axis as the imaginary part of $\operatorname{Im}(\omega) / k V_{l}$ is increased to positive values. Thus, the integrals in Eq. (57) can be done by deforming the velocity contour below the poles of interest. The poles and the residues are found numerically. 


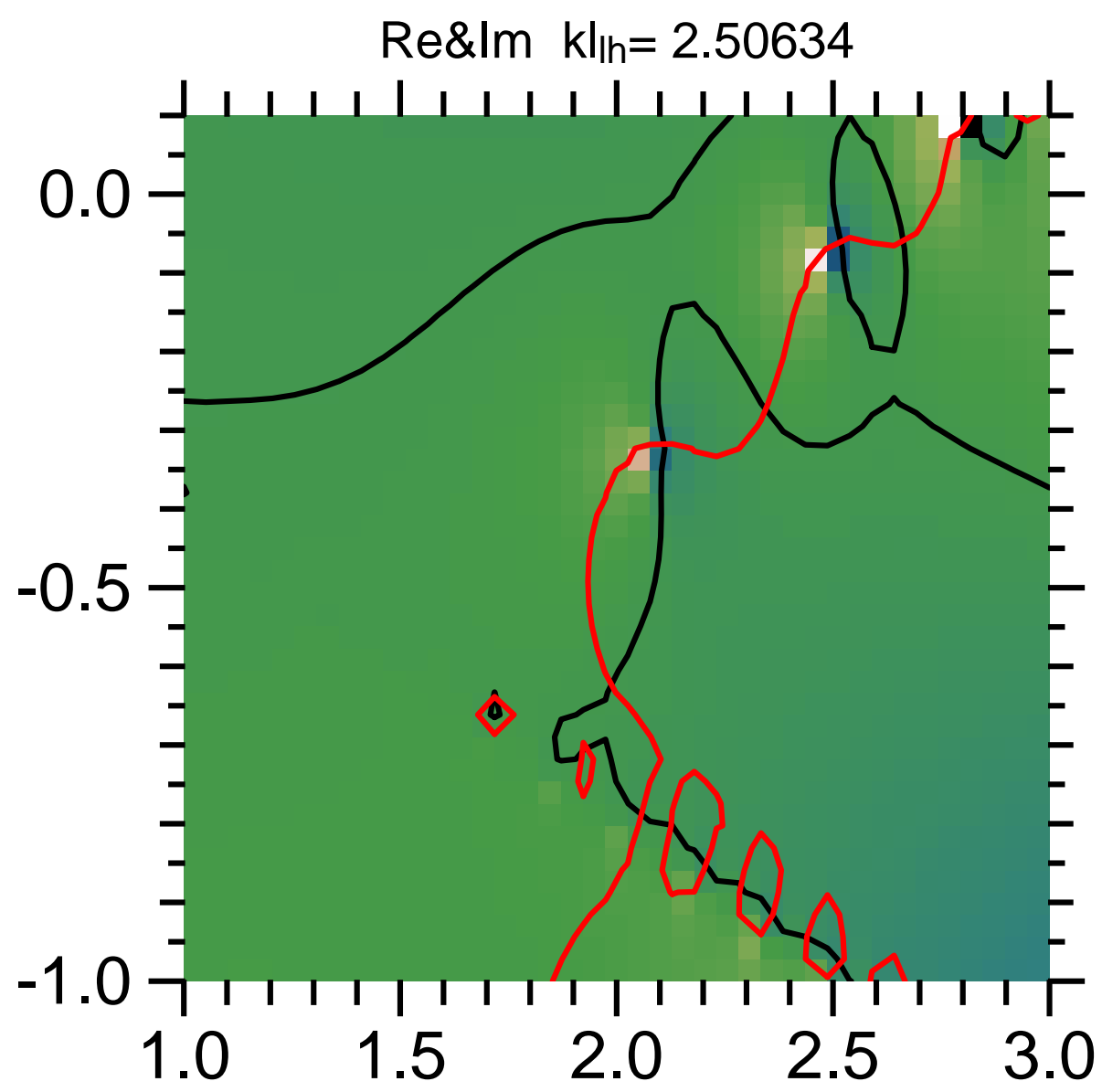

FIG. 15: The denominator of the integrand in Eq. (57) as a function of the complex velocity in the region where it has complex zeros. The black and red lines trace the contours where the real and imaginary part are zero respectively. The color plot shows the variation of the real part. The lines intersect both at zeros and simple poles. At poles, the color change from one side of the black line to the other indicates a change in sign of the real part of the denominator.

[1] J. D. Lindl ,P. Amendt ,R L. Berger ,S. G Glendinning, ,S H. Glenzer ,S. W. Haan ,R. L. Kauffman ,O. L. Landen, and L. J. Suter, "The physics basis for ignition using indirect-drive targets on the National Ignition Facility ," Phys. Plasmas 11, 339 (2004)

[2] D. H. Froula, L. Divol, and S. H. Glenzer, Phys. Rev. Lett. 88, 105003-1 (2002)

[3] E. A. Williams, R. L. Berger, R. P. Drake, A. M. Rubenchik, et al., Phys. Plasmas 2, 129 (1995). 
[4] H. X. Vu, J. M. Wallace, and B. Bezzerides, , Phys. Plasmas 1, 3542 (1994).

[5] S. H. Glenzer, C. A. Back, K. G. Estabrook, R. Wallace, et al., Phys. Rev. Lett. 77, 1496 (1996)

[6] J. D. Moody, private communication 2004

[7] R. M. Stevenson, L. J. Suter, K. Oades, W. L. Kruer, et al., Phys. Plasmas 00, 10000 (2004).

[8] E. M. Epperlein, R. W. Short, and A. Simon, PRE 49,2480 (1994).

[9] V. Yu. Bychenkov, W. Rozmus, and V. T. Tikhonchuk, PRE 51, 1400 (1995).

[10] D. E. Evans, Plasma Phys. 12, 573 (1970)

[11] B. D. Fried, R. B. White, and T. K. Samec, Phys. Fluids 14, 2388 (1971)

[12] J. A. Fejer, Can. J. Phys. 38, 1114 (1960)

[13] E. M. Epperlein, R. W. Short, and A. Simon. PRL69,1765 (1992).

[14] V. Yu. Bychenkov, J. Myatt, W. Rozmus, and V. T. Tikhonchuk, PRE 52, 6759 (1995).

[15] E. J. Valeo et al., BAPS 2002

[16] B. D. Fried and S. D. Conte, The Plasma Dispersion Function (Academic, New York, 1961)

[17] P. W. Rambo, S. C. Wilks, and W. L. Kruer, Phys. Rev. Lett. 79, 83 (1997)

[18] From this dispersion relation 52, one obtains the dispersion for electron damping of a single species ion acoustic wave with electron-ion collisions as given in Ref. [13] by letting $Z_{l} N_{l} \rightarrow N_{e}$, $Z_{h} N_{h} \rightarrow Z N_{i}, \nu_{l h} \rightarrow \nu_{e}$, and changing the sign of the $J_{4}$ term because of the charge in sign of the charge ratio, 


\section{External Distribution}

Plasma Research Laboratory, Australian National University, Australia

Professor I.R. Jones, Flinders University, Australia

Professor João Canalle, Instituto de Fisica DEQ/IF - UERJ, Brazil

Mr. Gerson O. Ludwig, Instituto Nacional de Pesquisas, Brazil

Dr. P.H. Sakanaka, Instituto Fisica, Brazil

The Librarian, Culham Laboratory, England

Mrs. S.A. Hutchinson, JET Library, England

Professor M.N. Bussac, Ecole Polytechnique, France

Librarian, Max-Planck-Institut für Plasmaphysik, Germany

Jolan Moldvai, Reports Library, Hungarian Academy of Sciences, Central Research Institute for Physics, Hungary

Dr. P. Kaw, Institute for Plasma Research, India

Ms. P.J. Pathak, Librarian, Institute for Plasma Research, India

Ms. Clelia De Palo, Associazione EURATOM-ENEA, Italy

Dr. G. Grosso, Instituto di Fisica del Plasma, Italy

Librarian, Naka Fusion Research Establishment, JAERI, Japan

Library, Laboratory for Complex Energy Processes, Institute for Advanced Study, Kyoto University, Japan

Research Information Center, National Institute for Fusion Science, Japan

Dr. O. Mitarai, Kyushu Tokai University, Japan

Dr. Jiangang Li, Institute of Plasma Physics, Chinese Academy of Sciences, People's Republic of China

Professor Yuping Huo, School of Physical Science and Technology, People's Republic of China

Library, Academia Sinica, Institute of Plasma Physics, People's Republic of China

Librarian, Institute of Physics, Chinese Academy of Sciences, People's Republic of China

Dr. S. Mirnov, TRINITI, Troitsk, Russian Federation, Russia

Dr. V.S. Strelkov, Kurchatov Institute, Russian Federation, Russia

Professor Peter Lukac, Katedra Fyziky Plazmy MFF UK, Mlynska dolina F-2, Komenskeho Univerzita, SK-842 15 Bratislava, Slovakia

Dr. G.S. Lee, Korea Basic Science Institute, South Korea

Institute for Plasma Research, University of Maryland, USA

Librarian, Fusion Energy Division, Oak Ridge National Laboratory, USA

Librarian, Institute of Fusion Studies, University of Texas, USA

Librarian, Magnetic Fusion Program, Lawrence Livermore National Laboratory, USA

Library, General Atomics, USA

Plasma Physics Group, Fusion Energy Research Program, University of California at San Diego, USA

Plasma Physics Library, Columbia University, USA

Alkesh Punjabi, Center for Fusion Research and Training, Hampton University, USA

Dr. W.M. Stacey, Fusion Research Center, Georgia Institute of Technology, USA

Dr. John Willis, U.S. Department of Energy, Office of Fusion Energy Sciences, USA

Mr. Paul H. Wright, Indianapolis, Indiana, USA 
The Princeton Plasma Physics Laboratory is operated by Princeton University under contract with the U.S. Department of Energy.

\author{
Information Services \\ Princeton Plasma Physics Laboratory \\ P.O. Box 451 \\ Princeton, NJ 08543
}

Phone: 609-243-2750

Fax: 609-243-2751

e-mail: pppl_info@pppl.gov

Internet Address: http://www.pppl.gov 